\title{
Quasi-elementary contractions of Fano manifolds
}

\author{
Cinzia Casagrande
}

\begin{abstract}
Let $X$ be a smooth complex Fano variety. We study 'quasi-elementary' contractions of fiber type of $X$, which are a natural generalization of elementary contractions of fiber type. If $f: X \rightarrow Y$ is such a contraction, then the Picard numbers satisfy $\rho_{X} \leqslant \rho_{Y}+\rho_{F}$, where $F$ is a general fiber of $f$. We show that, if $\operatorname{dim} Y \leqslant 3$ and $\rho_{Y} \geqslant 4$, then $Y$ is smooth and Fano; if moreover $\rho_{Y} \geqslant 6$, then $X$ is a product. This yields sharp bounds on $\rho_{X}$ when $\operatorname{dim} X=4$ and $X$ has a quasi-elementary contraction of fiber type, and other applications in higher dimensions.
\end{abstract}

\section{Contents}

1 Introduction . . . . . . . . . . . . . . . . . . . . 1429

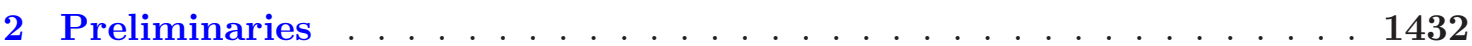

3 Quasi-elementary contractions . . . . . . . . . . . . . . . 1434

4 Existence of contractions after [BCD07] . . . . . . . . . . . . 1442

5 Fano manifolds with a quasi-elementary contraction onto a surface . 1445

6 Fano manifolds with a quasi-elementary contraction onto a 3-fold . . 1448

7 Applications and examples . . . . . . . . . . . . . . . 1455

References . . . . . . . . . . . . . . . . . . . 1458

\section{Introduction}

A Fano manifold is a smooth complex projective variety $X$ with ample anticanonical class. These varieties have a rich structure, and play an important role in higher dimensional geometry from the viewpoint of Mori theory, because they appear as fibers of Mori contractions of fiber type of smooth projective varieties.

After [Cam92, KMM92] we know that $X$ is rationally connected and simply connected. Moreover, smooth Fano varieties of dimension $n$ form a limited family, so they have only a finite number of possible topological types. However, in general, very little is known about their topological invariants.

In particular, we consider here the second Betti number $b_{2}$ of $X$, which coincides with the Picard number $\rho_{X}$.

Recall that a Del Pezzo surface $S$ has $\rho_{S} \leqslant 9$. Fano 3 -folds have been classified by Iskovskikh, Mori, and Mukai (see [IP99] and references therein). Thus we know that a Fano 3 -fold $X$ has $\rho_{X} \leqslant 10$. In fact, more is true: as soon as $\rho_{X} \geqslant 6, X$ is a product of a Del Pezzo surface with $\mathbb{P}^{1}$ (see [MM81, Theorem 2]).

Starting from dimension 4 , we do not have a bound on $\rho_{X}$. The known examples with largest Picard number are just products of Del Pezzo surfaces with Picard number 9, which gives $\rho_{X}=\frac{9}{2} n$.

Received 17 May 2007, accepted in final form 17 April 2008, published online 26 September 2008. 2000 Mathematics Subject Classification 14J45, 14E30.

Keywords: Fano varieties, Mori theory, Picard number.

This journal is (C) Foundation Compositio Mathematica 2008. 


\section{Casagrande}

Optimistically, one could think that Fano varieties with large Picard number are simpler, maybe a product of lower dimensional varieties. This would yield a linear bound (in the dimension $n$ ) for $\rho_{X}$; in fact one could expect precisely $\rho_{X} \leqslant \frac{9}{2} n$ (see [Deb03, p. 122]).

This is actually what happens in the toric case: if $X$ is a smooth toric Fano variety of dimension $n$, then $\rho_{X} \leqslant 2 n$, and equality holds if and only if $n$ is even and $X$ is $(S)^{n / 2}, S$ the blow-up of $\mathbb{P}^{2}$ in three non-collinear points (see [Cas06]).

Let us also recall that there is a class of Fano varieties for which a stronger linear bound on $\rho_{X}$ is expected. These are Fano varieties that do not contain curves of anticanonical degree 1, e.g. Fano varieties of index at least 2 . In this case it is expected that $\rho_{X} \leqslant n$, with equality only for $\left(\mathbb{P}^{1}\right)^{n}$. This is a generalization of a conjecture by Mukai, and has been proved in dimension $n \leqslant 5$ and in the toric case, see [BCDD03, ACO04, Cas06].

A strategy in this direction is to look for a contraction $f: X \rightarrow Y$ of fiber type on $X$, and try to bound $\rho_{X}$ in terms of $\rho_{Y}$ and $\rho_{F}$, where $F$ is a general fiber. There are (at least) two difficulties in this approach. First, $Y$ may not be Fano, so that we do not know how to bound $\rho_{Y}$. Second, surely $F$ is Fano, but in general $\rho_{F}$ is much smaller than $\rho_{X}-\rho_{Y}$ (for instance, any Del Pezzo surface $S$ admits a contraction $S \rightarrow \mathbb{P}^{1}$ with general fiber $\mathbb{P}^{1}$ ). This problem could be avoided by considering only elementary contractions of fiber type, for which $\rho_{X}=1+\rho_{Y}$. However, this is not very satisfactory, because we do not necessarily expect a Fano variety with large Picard number to have an elementary contraction of fiber type (think of a product of Del Pezzo surfaces).

This was our motivation to introduce the notion of 'quasi-elementary' contraction of fiber type. This is a contraction of fiber type as above, such that, if $i: F \hookrightarrow X$ is a general fiber, then the image of $i_{*}: \mathcal{N}_{1}(F) \rightarrow \mathcal{N}_{1}(X)$ contains all numerical classes of curves contracted by $f$ (see Definition 3.1). This implies that $\rho_{X} \leqslant \rho_{Y}+\rho_{F}$. In particular, any elementary contraction of fiber type is quasielementary. If a contraction is a smooth morphism (such as a projection $Y \times F \rightarrow Y$ ), then it is quasi-elementary (see Lemma 3.3).

We study several properties of quasi-elementary contractions of smooth Fano varieties, in particular when the target has small dimension. The following is our main result.

Theorem 1.1. Let $X$ be a smooth complex Fano variety, $f: X \rightarrow Y$ a quasi-elementary contraction of fiber type, and $F$ a general fiber.

(i) Suppose that $\operatorname{dim} Y=2$. Then $Y$ is a smooth Del Pezzo surface, $f$ is equidimensional, and $\rho_{X} \leqslant \rho_{Y}+\rho_{F} \leqslant 9+\rho_{F}$. If moreover $\rho_{Y} \geqslant 3$, then $X \cong Y \times F$ and $f$ is the projection on the first factor.

(ii) Suppose that $\operatorname{dim} Y=3$. Then $\rho_{X} \leqslant \rho_{Y}+\rho_{F} \leqslant 10+\rho_{F}$. If moreover $\rho_{Y} \geqslant 4$, then $Y$ is smooth and Fano. If $\rho_{Y} \geqslant 6$, then $X \cong S \times W$ and $Y \cong S \times \mathbb{P}^{1}$, where $S$ is a Del Pezzo surface, and $W$ a smooth Fano variety with a quasi-elementary contraction onto $\mathbb{P}^{1}$.

In the case $\operatorname{dim} X=3$, part (i) has been shown in [MM81, Proposition 8] under the more general assumption that $f: X \rightarrow Y$ is an equidimensional contraction onto a surface. See the end of $\S 7$ herein for examples concerning the sharpness of the statement.

The following are some applications to Fano 4-folds and 5-folds.

Corollary 1.2. Let $X$ be a smooth complex Fano 4-fold.

(i) If $X$ has a non-trivial quasi-elementary contraction of fiber type, then $\rho_{X} \leqslant 18$, with equality if and only if $X \cong S_{1} \times S_{2}$, where $S_{i}$ are Del Pezzo surfaces with $\rho_{S_{i}}=9$.

(ii) If $X$ has an elementary contraction onto a surface $S$ and $\rho_{X} \geqslant 4$, then $X \cong \mathbb{P}^{2} \times S$.

(iii) If $X$ has an elementary contraction onto a 3-fold and $\rho_{X} \geqslant 7$, then either $X \cong \mathbb{P}^{1} \times \mathbb{P}^{1} \times S$, or $X \cong \mathbb{F}_{1} \times S$, with $S$ a Del Pezzo surface. 


\section{QUASI-ELEMENTARY CONTRACTIONS OF FANO MANIFOLDS}

Corollary 1.3. Let $X$ be a smooth complex Fano 5-fold.

(i) If $X$ has two distinct elementary contractions of fiber type, then $\rho_{X} \leqslant 12$.

(ii) If $X$ has an elementary contraction onto $Y$ with $\operatorname{dim} Y \leqslant 3$, then $\rho_{X} \leqslant 11$.

(iii) Suppose that $X$ has an elementary contraction $f: X \rightarrow Y$ with $\operatorname{dim} Y=4$. If $X$ has another elementary contraction $\varphi$ of type $(3,0),(4,0),(4,1)$, or such that $f(\operatorname{Exc}(\varphi))=Y$, then $\rho_{X} \leqslant 12$.

Finally we give an application to Fano varieties with two quasi-elementary contractions.

Corollary 1.4. Let $X$ be a smooth complex Fano variety of dimension $n$. Let $f_{1}: X \rightarrow Y_{1}$ and $f_{2}: X \rightarrow Y_{2}$ be two quasi-elementary contractions of fiber type with $\mathrm{NE}\left(f_{1}\right) \cap \mathrm{NE}\left(f_{2}\right)=\{0\}$. Let $F_{i}$ be a general fiber of $f_{i}$. Then $\operatorname{dim} F_{1}+\operatorname{dim} F_{2} \leqslant n$, and moreover:

(i) $\operatorname{dim} F_{1}+\operatorname{dim} F_{2}=n$ implies that $\rho_{X} \leqslant \rho_{F_{1}}+\rho_{F_{2}}$;

(ii) $\operatorname{dim} F_{1}+\operatorname{dim} F_{2}=n-1$ implies that $\rho_{X} \leqslant \rho_{F_{1}}+\rho_{F_{2}}+1$;

(iii) $\operatorname{dim} F_{1}+\operatorname{dim} F_{2}=n-2$ implies that $\rho_{X} \leqslant \rho_{F_{1}}+\rho_{F_{2}}+9$; and

(iv) if $\operatorname{dim} F_{1}+\operatorname{dim} F_{2}=n-3$ and $f_{2}$ is elementary, then $\rho_{X} \leqslant \rho_{F_{1}}+11$.

If $f_{i}$ is elementary one can replace $\rho_{F_{i}}$ by 1 in the statement, see Remark 7.1. These corollaries should be compared to the following result by Wiśniewski, involving an arbitrary number of elementary contractions of fiber type.

Theorem 1.5 [Wiś91a, Theorem 2.2]. Let $X$ be a smooth complex Fano variety of dimension $n$. Suppose that $X$ has $k$ distinct elementary contractions of fiber type, and let $F_{1}, \ldots, F_{k}$ be the general fibers. Then $\sum_{i} \operatorname{dim} F_{i} \leqslant n$, and moreover:

(i) $\sum_{i} \operatorname{dim} F_{i}=n$ implies that $\rho_{X} \leqslant k$; and

(ii) $\sum_{i} \operatorname{dim} F_{i}=n-1$ implies that $\rho_{X} \leqslant k+1$.

In particular $k \leqslant n$, and if $k \geqslant n-1$ then $\rho_{X} \leqslant n$.

Our main tool is Mori theory; in particular we use many properties of contractions of Fano varieties shown in [Wiś91a].

In $\S 2$ we recall some basic notions and properties. In $\S 2.5$ we show that, when a variety $Y$ is the target of a contraction $f: X \rightarrow Y$ where $X$ is Fano, $Y$ shares with $X$ a good behavior with respect to Mori theory; see Lemma 2.6.

In $\S 3$ we define quasi-elementary contractions of fiber type $f: X \rightarrow Y$ and give some related properties and examples. We study the singularities of $Y$ in Lemma 3.10, generalizing results known in the elementary case and using in particular results from [ABW92]. Then in $\S 3.12$ we study elementary contractions of $Y$ by means of their liftings to $X$. This is a key ingredient in the proof of Theorem 1.1. Finally in $\S 3.15$ we show that if $X$ is Fano, $\operatorname{dim} Y \geqslant 3$ and $Y$ contains a prime divisor $D$ with $\rho_{D}=1$, then $\rho_{Y} \leqslant 3$, so $\rho_{X} \leqslant 3+\rho_{F}$ where $F$ is a general fiber of $f$. This is a generalization of results from [Tsu06] and [BCW02].

In $\S 4$ we apply results from [BCD07] to deduce that some contractions of $X$ always induce contractions of $Y$. This is needed in the proof of Theorem 1.1(i); moreover, it extends the applicability of Theorem $1.1(\operatorname{see} \S 7)$.

Section 5 contains the proof of Theorem 1.1(i), which relies on the results of $\S \S 3$ and 4 .

In $\S 6$ we show Theorem 1.1(ii). This is based on a detailed analysis of the possible elementary contractions of the target $Y$. We need the classification of smooth Fano 3-folds by Mori and Mukai, and we imitate the strategy for the classification of imprimitive smooth Fano 3-folds (see [MM81, IP99]) to get some results about the singular case. We also use the existence of a smoothing of a 


\section{Casagrande}

terminal Fano 3-fold $Z$ shown in [Nam97], and some relations among $Z$ and its smoothing shown in [JR06], see Lemma 6.10.

Finally in $\S 7$ we prove Corollaries $1.2,1.3$, and 1.4, and we give some other applications and related examples.

\section{Preliminaries}

We work over the field of complex numbers.

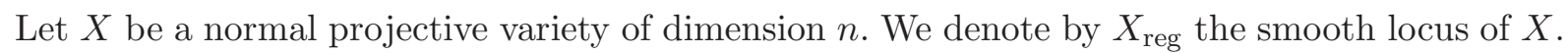

Let $\mathcal{N}_{1}(X)$ be the vector space of 1 -cycles in $X$ with real coefficients, modulo numerical equivalence. The dimension of $\mathcal{N}_{1}(X)$ is equal to the Picard number $\rho_{X}$ of $X$. Inside $\mathcal{N}_{1}(X)$ we denote by $\mathrm{NE}(X)$ the convex cone generated by classes of effective curves, and by $\overline{\mathrm{NE}}(X)$ its closure. If $C \subset X$ is a curve, its numerical class is $[C] \in \mathcal{N}_{1}(X)$.

If $Z$ is a closed subset of $X$, call $i: Z \hookrightarrow X$ the inclusion, and consider the linear map

$$
i_{*}: \mathcal{N}_{1}(Z) \longrightarrow \mathcal{N}_{1}(X)
$$

We denote by $\mathcal{N}_{1}(Z, X)$ the image of $i_{*}$ in $\mathcal{N}_{1}(X)$. Thus we have

$$
\operatorname{dim} \mathcal{N}_{1}(Z, X) \leqslant \rho_{Z} \quad \text { and } \quad \operatorname{dim} \mathcal{N}_{1}(Z, X) \leqslant \rho_{X}
$$

A contraction of $X$ is a surjective morphism with connected fibers $f: X \rightarrow Y$ onto a projective and normal variety $Y$. The push-forward of 1-cycles defined by $f$ gives a surjective linear map

$$
f_{*}: \mathcal{N}_{1}(X) \longrightarrow \mathcal{N}_{1}(Y)
$$

so that $\rho_{X}-\rho_{Y}=\operatorname{dim} \operatorname{ker} f_{*}$. We also consider the convex cone $\mathrm{NE}(f)$ in $\mathcal{N}_{1}(X)$ generated by classes of curves contracted by $f$, that is

$$
\mathrm{NE}(f)=\mathrm{NE}(X) \cap \operatorname{ker} f_{*} .
$$

The contraction $f$ is determined (up to isomorphism) by NE $(f)$, see [Deb01, Proposition 1.14]. We say that $f$ is of fiber type if $\operatorname{dim} Y<\operatorname{dim} X$, otherwise $f$ is birational. When $f$ is of fiber type, we say that $f$ is non-trivial if $\operatorname{dim} Y>0$. We denote by $\operatorname{Exc}(f)$ the exceptional locus of $f$, i.e. the locus where $f$ is not an isomorphism. We say that $f$ is divisorial if $\operatorname{Exc}(f)$ is a divisor, small if $\operatorname{Exc}(f)$ has codimension bigger than 1. More generally we say that $f$ is of type $(a, b)$ if $\operatorname{dim} \operatorname{Exc}(f)=a$ and $\operatorname{dim} f(\operatorname{Exc}(f))=b$. Finally $f$ is elementary if $\rho_{X}-\rho_{Y}=1$.

We will need to work with singular varieties; we refer the reader to [Deb01, KM98] for the definitions and properties of terminal and canonical singularities. We say that $X$ is $\mathbb{Q}$-factorial if every Weil divisor is $\mathbb{Q}$-Cartier.

Suppose that $X$ has canonical singularities (in particular $K_{X}$ is $\mathbb{Q}$-Cartier). We say that $f: X \rightarrow$ $Y$ is a Mori contraction if it is a contraction and moreover $-K_{X} \cdot C>0$ for every curve $C \subset X$ contracted by $f$. We recall two important properties of Mori contractions:

(2.1) $\operatorname{dim} \mathrm{NE}(f)=\operatorname{dim} \operatorname{ker} f_{*}=\rho_{X}-\rho_{Y}$, namely ker $f_{*}$ is the linear subspace generated by $\mathrm{NE}(f)$;

(2.2) for any $L \in \operatorname{Pic} X$ one has $L \in f^{*}(\operatorname{Pic} Y)$ if and only if $L \cdot C=0$ for every curve $C \subset X$ contracted by $f$.

(See [KM98, Theorem 3.7(4)] for the second statement, which implies the first one.)

Suppose that $\mathrm{NE}(X)$ is closed and polyhedral. By a face of $\mathrm{NE}(X)$ we just mean a face in the geometrical sense. For any contraction $f$ of $X, \mathrm{NE}(f)$ is a face of $\mathrm{NE}(X)$. An extremal ray is a one-dimensional face, with no assumptions on the intersection of $K_{X}$ with its elements. We will use greek letters $\alpha, \beta$, etc. to denote faces of $\operatorname{NE}(X)$. If $\alpha$ is an extremal ray and $D$ a $\mathbb{Q}$-Cartier divisor 


\section{QUASI-ELEMENTARY CONTRACTIONS OF FANO MANIFOLDS}

on $X$, we will say that $D \cdot \alpha>0, D \cdot \alpha=0$, or $D \cdot \alpha<0$ if respectively $D \cdot v>0, D \cdot v=0$, or $D \cdot v<0$ for a non-zero element $v \in \alpha$. We denote by $\operatorname{Locus}(\alpha) \subseteq X$ the union of all curves in $X$ whose numerical class is in $\alpha$.

Let $X$ be a projective variety with canonical singularities and $K_{X}$ Cartier. We say that $X$ is Fano if $-K_{X}$ is ample. If so, the cone $\mathrm{NE}(X)$ is closed and polyhedral, and any contraction of $X$ is a Mori contraction. Moreover, for any face $\alpha$ of $\mathrm{NE}(X)$ there exists a contraction $f$ of $X$ such that $\alpha=\mathrm{NE}(f)$. This follows from the contraction theorem, see [KM98, Theorem 3.7].

Remark 2.3. Let $X$ and $Y$ be factorial projective varieties, and $\sigma: X \rightarrow Y$ the blow-up of a smooth

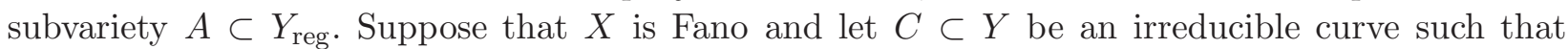
$C \not \subset A$ and $C \cap A \neq \emptyset$. Let $\widetilde{C}$ be the proper transform of $C$ in $X$. Then

$$
-K_{X} \cdot \widetilde{C}<-K_{Y} \cdot C
$$

in particular $-K_{Y} \cdot C \geqslant 2$.

In fact, if $E=\operatorname{Exc}(\sigma)$, we have $\widetilde{C} \nsubseteq E$ and $\widetilde{C} \cap E \neq \emptyset$, so $\widetilde{C} \cdot E>0$. Moreover $K_{X}=\sigma^{*}\left(K_{Y}\right)+a E$ where $a=\operatorname{codim} A-1$, so

$$
-K_{X} \cdot \widetilde{C}=-K_{Y} \cdot C-a E \cdot \widetilde{C}<-K_{Y} \cdot C .
$$

Remark 2.4. Let $X$ be a normal and $\mathbb{Q}$-factorial projective variety and $f: X \rightarrow Y$ an elementary divisorial contraction. Then $\operatorname{Exc}(f)$ is an irreducible divisor and $\operatorname{Exc}(f) \cdot \mathrm{NE}(f)<0$.

In fact, let $E$ be an irreducible component of $\operatorname{Exc}(f)$; then $E \cdot \mathrm{NE}(f)<0$ (see for instance [KM98, Lemma 3.39] applied to $B=-E$ ). Now if $C \subset X$ is an irreducible curve contracted by $f$, we have $E \cdot C<0$, thus $C \subseteq E$. Hence $E=\operatorname{Exc}(f)$.

\subsection{Targets of contractions of smooth Fano varieties}

Let $X$ be a smooth Fano variety and $f: X \rightarrow Y$ a contraction. Consider the push-forward $f_{*}: \mathcal{N}_{1}(X)$ $\rightarrow \mathcal{N}_{1}(Y)$. We observe that

$$
f_{*}(\mathrm{NE}(X))=\mathrm{NE}(Y),
$$

namely $\mathrm{NE}(Y)$ is the linear projection of $\mathrm{NE}(X)$ from the face $\mathrm{NE}(f)$. This simple remark implies many properties of $\mathrm{NE}(Y)$. For instance, it is closed and polyhedral, since $\mathrm{NE}(X)$ is. Moreover, faces of $\mathrm{NE}(Y)$ are in bijection (via $f_{*}$ ) with faces of $\mathrm{NE}(X)$ containing $\mathrm{NE}(f)$. In fact this description is the same as the one involving the 'star of a cone' in toric geometry, see [Ful93, p. 52].

Let us consider a face $\alpha$ of $\mathrm{NE}(Y)$, and let $\widehat{\alpha}$ be the unique face of $\mathrm{NE}(X)$ containing $\mathrm{NE}(f)$ and such that $f_{*}(\widehat{\alpha})=\alpha$. Then $\operatorname{dim} \widehat{\alpha}=\operatorname{dim} \alpha+\operatorname{dim} \mathrm{NE}(f)$. Since $\mathrm{NE}(f)$ is a face of $\widehat{\alpha}$, we can choose another face $\widetilde{\alpha}$ of $\widehat{\alpha}$ with the properties:

$$
\operatorname{dim} \widetilde{\alpha}=\operatorname{dim} \alpha \quad \text { and } \quad \widetilde{\alpha} \cap \mathrm{NE}(f)=\{0\} .
$$

Observe that the choice of $\widetilde{\alpha}$ will not be unique in general, and that it can very well be $\widetilde{\alpha}+\mathrm{NE}(f) \subsetneq \widehat{\alpha}$.

When $\alpha$ is an extremal ray, $\widetilde{\alpha}$ is an extremal ray of $\operatorname{NE}(X)$, and $f_{*}(\widetilde{\alpha})=\alpha$. There is a rational curve $C \subset X$ such that $[C] \in \widetilde{\alpha}$, hence $f(C)$ is a rational curve in $Y$ with numerical class in $\alpha$.

Since $X$ is Fano, there exist contractions $\varphi: X \rightarrow W$ and $h: X \rightarrow Z$ such that $\widetilde{\alpha}=\mathrm{NE}(\varphi)$ and $\widehat{\alpha}=\mathrm{NE}(h)$, as shown below.

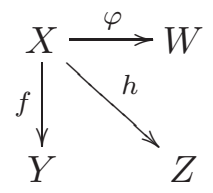




\section{Casagrande}

Now by rigidity (see for instance [Deb01, Lemma 1.15]) there exist contractions $\psi: Y \rightarrow Z$ and $g: W \rightarrow Z$ that make the following diagram commute.

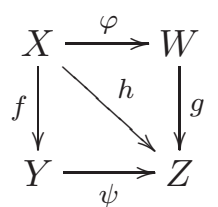

It is not difficult to check that $\operatorname{NE}(\psi)=\alpha$ and that $\operatorname{dim} \operatorname{ker} \psi_{*}=\operatorname{dim} \alpha$. We will say that $\varphi: X \rightarrow W$ is a lifting of $\psi$. Summing up, we have proved the following result.

Lemma 2.6. Let $X$ be a smooth Fano variety and $f: X \rightarrow Y$ a contraction. Then $\mathrm{NE}(Y)$ is a closed polyhedral cone, and every extremal ray contains the class of some rational curve.

Moreover for every face $\alpha$ of $\mathrm{NE}(Y)$ there exists a contraction $\psi: Y \rightarrow Z$ such that $\mathrm{NE}(\psi)=\alpha$ and $\rho_{Y}-\rho_{Z}=\operatorname{dim} \alpha$.

\section{Quasi-elementary contractions}

Let $X$ be a smooth variety and $f: X \rightarrow Y$ a Mori contraction of fiber type. Recall that

$$
\rho_{X}-\rho_{Y}=\operatorname{dim} \mathrm{NE}(f)=\operatorname{dim} \operatorname{ker} f_{*} .
$$

Let $F$ be a general fiber and consider $\mathcal{N}_{1}(F, X) \subseteq \mathcal{N}_{1}(X)$. We have

$$
\mathcal{N}_{1}(F, X) \subseteq \operatorname{ker} f_{*},
$$

hence $\operatorname{dim} \mathcal{N}_{1}(F, X) \leqslant \rho_{X}-\rho_{Y}$.

DeFinition 3.1. We say that $f$ is quasi-elementary if

$$
\mathcal{N}_{1}(F, X)=\operatorname{ker} f_{*},
$$

equivalently if $\operatorname{dim} \mathcal{N}_{1}(F, X)=\rho_{X}-\rho_{Y}$. Since $\operatorname{dim} \mathcal{N}_{1}(F, X) \leqslant \rho_{F}$, if $f$ is quasi-elementary we get

$$
\rho_{X} \leqslant \rho_{Y}+\rho_{F} .
$$

Example 3.2. Let $f: X \rightarrow Y$ be a Mori contraction of fiber type.

(a) If $f$ is elementary, then it is also quasi-elementary. This is because $1 \leqslant \operatorname{dim} \mathcal{N}_{1}(F, X) \leqslant$ $\operatorname{dim} \operatorname{ker} f_{*}=1$.

(b) Suppose that $\operatorname{dim} X-\operatorname{dim} Y=1$. Then $f$ is quasi-elementary if and only if it is elementary.

(c) Suppose that $X$ is Fano and $f$ is quasi-elementary. If $\psi: Y \rightarrow Z$ is an elementary contraction of fiber type, then the composition $\psi \circ f: X \rightarrow Z$ is quasi-elementary. In fact, since $\psi$ is elementary, we have $\operatorname{dim} \operatorname{ker}(\psi \circ f)_{*}=\operatorname{dim} \operatorname{ker} f_{*}+1$. Let $G$ be a general fiber of $\psi \circ f$, then $G \supset F$, hence $\operatorname{ker}(\psi \circ f)_{*} \supseteq \mathcal{N}_{1}(G, X) \supseteq \mathcal{N}_{1}(F, X)=\operatorname{ker} f_{*}$. Choosing a curve $C \subset G$ not contracted by $f$ shows that $\mathcal{N}_{1}(G, X) \supsetneq \mathcal{N}_{1}(F, X)$, so $\mathcal{N}_{1}(G, X)=\operatorname{ker}(\psi \circ f)_{*}$ and $\psi \circ f$ is quasi-elementary.

Let us show that the notion of quasi-elementary is related to smoothness.

Lemma 3.3. Let $X$ be a smooth variety and $f: X \rightarrow Y$ a Mori contraction of fiber type. Let $Y_{0} \subseteq Y_{\text {reg }}$ be an open subset over which $f$ is smooth, and set $X_{0}:=f^{-1}\left(Y_{0}\right)$. If $\operatorname{codim}\left(X \backslash X_{0}\right) \geqslant 2$ and $Y$ is $\mathbb{Q}$-factorial, then $f$ is quasi-elementary.

We remark that the converse to Lemma 3.3 does not hold (take, for instance, a smooth Fano 3 -fold $X$ with an elementary contraction $X \rightarrow \mathbb{P}^{1}$ that is not a smooth morphism). Moreover the hypothesis of $\mathbb{Q}$-factoriality on $Y$ is necessary, see the contraction $f: V \rightarrow Y$ in Example 7.9. 


\section{QUASI-ELEMENTARY CONTRACTIONS OF FANO MANIFOLDS}

Proof. Set $f_{0}:=f_{\mid X_{0}}: X_{0} \rightarrow Y_{0}$. Following the notation of [KMM87, $\left.\S 0-1\right]$, consider

$$
\mathrm{NE}\left(X_{0} / Y_{0}\right) \subset \mathcal{N}_{1}\left(X_{0} / Y_{0}\right) .
$$

Since $f_{0}$ is a Mori contraction, $\mathrm{NE}\left(X_{0} / Y_{0}\right)$ is closed and polyhedral by the relative version of the cone theorem, see [KMM87, Theorem 4-2-1].

On the other hand $\mathcal{N}_{1}(X / Y)=\operatorname{ker} f_{*} \subseteq \mathcal{N}_{1}(X)$, and the inclusion $X_{0} \hookrightarrow X$ induces a natural injective homomorphism

$$
\mathcal{N}_{1}\left(X_{0} / Y_{0}\right) \hookrightarrow \operatorname{ker} f_{*} .
$$

With a slight abuse of notation, we will consider $\mathcal{N}_{1}\left(X_{0} / Y_{0}\right)$ as a subspace of $\mathcal{N}_{1}(X)$.

For every $y \in Y_{0}$ we have $i_{*}\left(\mathrm{NE}\left(F_{y}\right)\right) \subseteq \mathrm{NE}\left(X_{0} / Y_{0}\right)$. Consider an extremal ray $\alpha$ of $\mathrm{NE}\left(X_{0} / Y_{0}\right)$. Then [Wiś91b, Proposition 1.3] says that Locus $(\alpha)$ dominates $Y_{0}$ via $f$, hence $\alpha \subseteq i_{*}\left(\mathrm{NE}\left(F_{y}\right)\right)$ for every $y \in Y_{0}$. Repeating this for every extremal ray of $\mathrm{NE}\left(X_{0} / Y_{0}\right)$, we get

$$
i_{*}\left(\mathrm{NE}\left(F_{y}\right)\right)=\mathrm{NE}\left(X_{0} / Y_{0}\right)
$$

for every $y \in Y_{0}$, in particular

$$
\mathcal{N}_{1}\left(F_{y}, X\right)=\mathcal{N}_{1}\left(X_{0} / Y_{0}\right) \subseteq \operatorname{ker} f_{*} \subseteq \mathcal{N}_{1}(X) .
$$

The homomorphism dual to the inclusion $\mathcal{N}_{1}\left(X_{0} / Y_{0}\right) \hookrightarrow \operatorname{ker} f_{*}$ is

$$
r: \frac{(\operatorname{Pic} X) \otimes \mathbb{R}}{f^{*}(\operatorname{Pic} Y) \otimes \mathbb{R}} \longrightarrow \frac{\left(\operatorname{Pic} X_{0}\right) \otimes \mathbb{R}}{f_{0}^{*}\left(\operatorname{Pic} Y_{0}\right) \otimes \mathbb{R}}
$$

induced by the restriction Pic $X \rightarrow \operatorname{Pic} X_{0}$ (see [KMM87, $\S 0-1$ and Lemma 3-2-5(2)]). Clearly $r$ is surjective.

Let $L \in \operatorname{Pic} X$ be such that $L_{\mid X_{0}}=f_{0}^{*}\left(M_{0}\right)$ for some $M_{0} \in \operatorname{Pic} Y_{0}$. Since $Y$ is $\mathbb{Q}$-factorial, there exists $M \in \operatorname{Pic} Y$ such that $M_{\mid Y_{0}}=M_{0}^{\otimes l}, l \in \mathbb{Z}_{\geqslant 1}$. Then $L^{\otimes l} \otimes f^{*}(M)^{\otimes(-1)}$ is trivial on $X_{0}$, and by our hypothesis trivial on $X$. Thus $r$ is an isomorphism. Dually, this is gives $\mathcal{N}_{1}\left(F_{y}, X\right)=$ $\mathcal{N}_{1}\left(X_{0} / Y_{0}\right)=\operatorname{ker} f_{*}$, so $f$ is quasi-elementary.

Remark 3.5. Observe that (3.4) shows that $\mathcal{N}_{1}\left(F_{y}, X\right)$ does not depend on $y \in Y_{0}$, so that the condition $\mathcal{N}_{1}\left(F_{y}, X\right)=\operatorname{ker} f_{*}$ can be checked for an arbitrary $y \in Y_{0}$.

Remark 3.6. If $f: X \rightarrow Y$ is quasi-elementary, then $\mathcal{N}_{1}\left(F_{0}, X\right)=\operatorname{ker} f_{*}$ for every fiber $F_{0}$ of $f$ (with the reduced structure).

In fact we have $\mathcal{N}_{1}\left(F_{0}, X\right) \subseteq \operatorname{ker} f_{*}$. Moreover if $f_{0}: X_{0} \rightarrow Y_{0}$ is as in the proof of Lemma 3.3, $\mathrm{NE}\left(X_{0} / Y_{0}\right)$ has dimension $\rho_{X}-\rho_{Y}$. For any fixed extremal ray $\alpha$ of $\mathrm{NE}\left(X_{0} / Y_{0}\right)$, we know by [Wiś91b, Proposition 1.3] that $\operatorname{Locus}(\alpha)$ dominates $Y_{0}$. Then taking a family of curves whose class is in $\alpha$ and their degenerations, we see that $\alpha \subset \mathcal{N}_{1}\left(F_{0}, X\right)$. This implies that $\mathcal{N}_{1}\left(F_{0}, X\right)=\operatorname{ker} f_{*}$.

Remark 3.7. Suppose that $X$ is smooth and Fano, $f: X \rightarrow Y$ a contraction of fiber type, and $F$ a general fiber. In general the push-forward $i_{*}: \mathcal{N}_{1}(F) \rightarrow \mathcal{N}_{1}(X)$ does not need to be injective: for instance, there are smooth Fano 3-folds that have an elementary contraction onto $\mathbb{P}^{1}$, with fibers Del Pezzo surfaces with $\rho>1$. This is related to the monodromy of the fibration $f$.

Consider an open subset $Y_{0}$ as in Lemma 3.3 and let $y \in Y_{0}$. The dimension of $\mathcal{N}_{1}\left(F_{y}, X\right)$ is equal to the dimension of the image of the restriction

$$
(\operatorname{Pic} X) \otimes \mathbb{Q}=H^{2}(X, \mathbb{Q}) \longrightarrow H^{2}\left(F_{y}, \mathbb{Q}\right)=\left(\operatorname{Pic} F_{y}\right) \otimes \mathbb{Q} .
$$

In turn this is equal to the dimension of the linear subspace of $H^{2}\left(F_{y}, \mathbb{Q}\right)$ which is invariant for the monodromy action of $\pi_{1}\left(Y_{0}, y\right)$ (see for instance [Voi02, ch. 15]). Hence $\operatorname{dim} \mathcal{N}_{1}\left(F_{y}, X\right)=\rho_{F}$ if and only if the monodromy action is trivial. 


\section{Casagrande}

Example 3.8. Let $X$ be a smooth Fano variety, $f: X \rightarrow Y$ a non-trivial contraction of fiber type with $Y$ smooth, and $F$ a general fiber. Suppose that $f$ is smooth outside a finite number of points of $Y$, and that there are no fibers of codimension 1. Then $Y$ is Fano, $f$ is quasi-elementary, and $\rho_{X}=\rho_{Y}+\rho_{F}$.

In fact $Y$ is Fano by [Miy93, Theorem 3]; in particular it is simply connected, and $\operatorname{dim} Y \geqslant 2$. Then $Y \backslash\left\{y_{1}, \ldots, y_{m}\right\}$ stays simply connected, so the monodromy action on $H^{2}(F, \mathbb{Q})$ is trivial, and $\operatorname{dim} \mathcal{N}_{1}(F, X)=\rho_{F}$. On the other hand $f$ is quasi-elementary by Lemma 3.3 , so $\rho_{X}=$ $\operatorname{dim} \mathcal{N}_{1}(F, X)+\rho_{Y}=\rho_{F}+\rho_{Y}$.

The following two lemmas give some basic properties of quasi-elementary contractions.

Lemma 3.9. Let $X$ be a smooth variety and $f: X \rightarrow Y$ a quasi-elementary Mori contraction of fiber type.

(i) If $D$ is a prime divisor in $X$ such that $f(D) \subsetneq Y$, then $f(D)$ is a Cartier divisor, and $D=$ $f^{*}(f(D))$.

(ii) The locus where $f$ is not equidimensional has codimension at least 3 in $Y$.

Proof. Since $f$ is quasi-elementary and $D$ is disjoint from the general fiber, we have $D \cdot C=0$ for every curve $C \subset X$ contracted by $f$. By property (2.2) of Mori contractions, there exists an effective Cartier divisor $D^{\prime}$ on $Y$ such that $D=f^{*}\left(D^{\prime}\right)$. Moreover $D^{\prime}$ is supported on $f(D)$, so there exists $m \in \mathbb{Z}_{>0}$ such that $D^{\prime}=m f(D)$.

Set $U:=f^{-1}\left(Y_{\text {reg }}\right)$ and observe that $X \backslash U$ has codimension at least 2 in $X$. In $Y_{\text {reg }}$ the intersection $f(D) \cap Y_{\text {reg }}$ is a (non-trivial) Cartier divisor and $D_{\mid U}=\left(f_{\mid U}\right)^{*}(m f(D))_{\mid Y_{\text {reg }}}=m\left(f_{\mid U}\right)^{*}(f(D) \cap$ $\left.Y_{\text {reg }}\right)$. Since $X$ is smooth, there exists an effective divisor $D^{\prime \prime}$ in $X$ such that $D_{\mid U}^{\prime \prime}=\left(f_{\mid U}\right)^{*}(f(D) \cap$ $\left.Y_{\text {reg }}\right)$. Then $D_{\mid U}=m D_{\mid U}^{\prime \prime}$, so $D=m D^{\prime \prime}$ and $m=1$ because $D$ is prime.

Let us show part (ii). Let $K \subset Y$ be an irreducible closed subset such that the general fiber of $f$ over $K$ has dimension at least $\operatorname{dim} X-\operatorname{dim} Y+1$. Then

$$
\operatorname{dim} X-1 \geqslant \operatorname{dim} f^{-1}(K) \geqslant \operatorname{dim} X-\operatorname{dim} Y+1+\operatorname{dim} K,
$$

so $\operatorname{dim} K \leqslant \operatorname{dim} Y-2$. If by contradiction $\operatorname{dim} K=\operatorname{dim} Y-2$, then $\operatorname{dim} f^{-1}(K) \geqslant \operatorname{dim} X-1$. Consider a prime divisor $D$ contained in $f^{-1}(K)$ : we have $f(D) \subseteq K$, which contradicts (i).

We generalize to quasi-elementary contractions some known properties of elementary contractions of fiber type. In particular part (ii) below is shown in [ABW92] in the elementary case.

Lemma 3.10. Let $f: X \rightarrow Y$ be a Mori contraction of fiber type, with $X$ smooth.

(i) If $f$ is quasi-elementary, then $Y$ is factorial and has canonical singularities.

(ii) If $Y$ is a surface and $f$ is equidimensional, then $Y$ is smooth.

(iii) If $Y$ is a surface and $f$ is quasi-elementary, then $f$ is equidimensional and $Y$ is smooth.

(iv) If $\operatorname{dim} Y=3$ and $f$ is quasi-elementary, then $Y$ has isolated singularities.

Proof. (i) Let $D$ be a prime Weil divisor in $Y$ and let $D^{\prime}$ be a prime divisor in $X$ such that $f\left(D^{\prime}\right)=D$. Then Lemma 3.9(i) yields that $D$ is Cartier.

Thus $Y$ is factorial, in particular $K_{Y}$ is Cartier. It is known that $Y$ has rational singularities, see [Kol86, Corollary 7.4]. Then $Y$ also has canonical singularities, see [KM98, Corollary 5.24].

(ii) When $f$ is elementary, this is [ABW92, Proposition 1.4.1]. In general, observe that $Y$ is a normal surface with rational singularities; in particular it is $\mathbb{Q}$-factorial and has isolated singularities.

We want to show that in fact $Y$ has quotient singularities, using results from [Wat80] and [FZ03]. More precisely, let $y_{0} \in Y$ be a singular point. In [Wat80, Definition 1.4] and [FZ03, Definition 1.25] 


\section{QUASI-ELEMENTARY CONTRACTIONS OF FANO MANIFOLDS}

one can find the definition of the plurigenera $\delta_{m}\left(Y, y_{0}\right)$ of $Y$ in $y_{0}$, for $m \in \mathbb{Z}_{>0}$. Since $f$ is equidimensional, then it is 'non-degenerate' in the sense of [FZ03, Definition 1.14]. Then [FZ03, Corollary 1.27] gives $\delta_{m}\left(Y, y_{0}\right)=0$ for every $m \in \mathbb{Z}_{>0}$. This is equivalent to saying that $y_{0}$ is a quotient singularity by [Wat80, Theorem 3.9].

Hence $Y$ has quotient singularities, and we can apply [ABW92, Proposition 1.4] to deduce that $Y$ is actually smooth. Observe that in [ABW92] the contraction $f$ is assumed to be elementary, but the proof works word for word in the case of an equidimensional Mori contraction. Let us also remark that, by the definition of quotient singularities, we can cover $Y$ by open subsets in the complex topology which are quotients of an open subset of $\mathbb{C}^{2}$ by a finite group. Hence in the proof of [ABW92, Proposition 1.4] one has actually to work in the analytic category; however everything works in the same way.

(iii) This follows immediately from Lemma 3.9 and part (ii).

(iv) By Lemma 3.9, $f$ can have at most isolated fibers of dimension $n-2$. Let $S \subset Y$ be a general hyperplane section and $D:=f^{-1}(S)$. Then $S$ is a normal surface (see [KM98, Lemma 5.30]) and $f_{\mid D}: D \rightarrow S$ is equidimensional. Moreover $D$ is general in a base point free linear system, so it is smooth. Since $D$ is disjoint from the general fiber of $f$ and $f$ is quasi-elementary, we have $D \cdot C=0$ for every curve $C \subset X$ contracted by $f$. In particular, if $C \subset D$ we get

$$
-K_{D} \cdot C=-K_{X} \cdot C>0,
$$

so $f_{\mid D}$ is an equidimensional Mori contraction. Then $S$ is smooth by part (ii), and this yields $\operatorname{dim} \operatorname{Sing} Y=0$.

Thus the target of a quasi-elementary contraction has reasonable singularities. The following simple remark will be very useful.

Remark 3.11. Let $X$ be a smooth Fano variety and $f: X \rightarrow Y$ a quasi-elementary contraction, so that $Y$ is factorial and has canonical singularities by Lemma 3.10. Then $Y$ is Fano if and only if $-K_{Y} \cdot \alpha>0$ for every extremal ray $\alpha$ of $\mathrm{NE}(Y)$. Equivalently, $Y$ is Fano if and only if every elementary contraction $\psi: Y \rightarrow Z$ is a Mori contraction.

This is a straightforward consequence of Lemma 2.6.

\subsection{Elementary contractions of the target}

Let $X$ be a smooth projective variety and $f: X \rightarrow Y$ a quasi-elementary Mori contraction of fiber type. Recall that $Y$ is factorial with canonical singularities by Lemma 3.10.

Let $\psi: Y \rightarrow Z$ be a contraction. We say that $\psi$ has a lifting if there exist a Mori contraction $\varphi: X \rightarrow Y$ such that $\mathrm{NE}(\varphi) \cap \mathrm{NE}(f)=\{0\}, \rho_{X}-\rho_{W}=\rho_{Y}-\rho_{Z}$, and a commutative diagram as below.

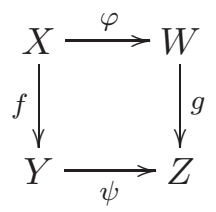

We will also say that $\varphi$ is a lifting of $\psi$.

Notice that $\psi$ is elementary if and only if $\varphi$ is elementary. We are interested in comparing properties of $\varphi$ and $\psi$.

When $X$ is Fano, any contraction $\psi: Y \rightarrow Z$ has a lifting, as explained in $\S 2.5$. Conversely, in Theorems 4.1 and 4.4 we will give conditions on a Mori contraction $\varphi: X \rightarrow W$ to be a lifting of some $\psi$. 


\section{Casagrande}

Theorem 3.14. Let $X$ be a smooth projective variety and $f: X \rightarrow Y$ a quasi-elementary Mori contraction of fiber type.

Let $\psi: Y \rightarrow Z$ be a birational elementary contraction with fibers of dimension at most 1 . Assume that $\psi$ has a lifting $\varphi: X \rightarrow W$ as in (3.13). Then the following hold:

(i) $W$ is smooth and $\varphi$ is the blow-up of a smooth subvariety of codimension 2;

(ii) $g$ is a quasi-elementary Mori contraction and $Z$ is factorial with canonical singularities;

(iii) $\psi$ is divisorial, $\operatorname{Exc}(\varphi)=f^{*}(\operatorname{Exc}(\psi))$, and $-K_{Y} \cdot \operatorname{NE}(\psi) \geqslant 0$;

(iv) if $\operatorname{dim} Y=3$, then $\psi$ is the blow-up of a smooth curve $C \subset Z_{\text {reg }}$; and

(v) if $\operatorname{dim} Y=2$, then

(v.a) $Y$ and $Z$ are smooth, $\psi$ is the blow-up of $z_{0} \in Z$, and $g$ has smooth fiber $F_{0}$ over $z_{0}$;

(v.b) $\varphi$ is the blow-up of $F_{0}, \operatorname{Exc}(\varphi) \cong \mathbb{P}^{1} \times F_{0}$, and $f_{\mid \operatorname{Exc}(\varphi)}, \varphi_{\mid \operatorname{Exc}(\varphi)}$ are the two projections;

(v.c) if $X$ is Fano, then $W$ is Fano.

Proof. Let $F$ be a non-trivial fiber of $\varphi$. Then $f$ is finite on $F$ and $f(F)$ is contained in a non-trivial fiber of $\psi$, hence $f(F) \subseteq \operatorname{Exc}(\psi)$ and $\operatorname{dim} F=1$. Since $\operatorname{Exc}(\varphi)$ is covered by non-trivial fibers of $\varphi$, we get $f(\operatorname{Exc}(\varphi)) \subseteq \operatorname{Exc}(\psi)$. In particular, $\varphi$ is birational with fibers of dimension at most 1 , so [Wiś91a, Theorem 1.2] yields that $W$ is smooth and $\varphi$ is the blow-up of a smooth subvariety of codimension 2.

Let $E \subset X$ be the exceptional divisor of $\varphi$, then $f(E)$ is contained in $\operatorname{Exc}(\psi)$ and it is a divisor by Lemma 3.9(i). Hence $\psi$ is elementary and divisorial, and $\operatorname{Exc}(\psi)$ is irreducible by Remark 2.4, that is $\operatorname{Exc}(\psi)=f(E)$. Then again Lemma 3.9(i) gives $E=f^{*}(\operatorname{Exc}(\psi))$.

We have

$$
K_{X}=\varphi^{*}\left(K_{W}\right)+E .
$$

Let $C$ be an irreducible curve contracted by $g$ and $\widetilde{C}$ an irreducible curve in $X$ such that $\varphi(\widetilde{C})=C$. Then $\varphi_{*}(\widetilde{C})=m C$ with $m \in \mathbb{Z}_{>0}$. Moreover $f(\widetilde{C})$ is a point, so $\widetilde{C} \cdot E=0$. Then

$$
m\left(-K_{W} \cdot C\right)=-K_{W} \cdot \varphi_{*}(\widetilde{C})=\left(-K_{X}-E\right) \cdot \widetilde{C}=-K_{X} \cdot \widetilde{C}>0,
$$

so $g$ is a Mori contraction.

We have $\operatorname{dim} \operatorname{ker} f_{*}=\rho_{X}-\rho_{Y}=\rho_{W}-\rho_{Z}=\operatorname{dim} \operatorname{ker} g_{*}$. Moreover $\operatorname{NE}(\varphi)$ is an extremal ray of $\mathrm{NE}(X)$ not contained in $\mathrm{NE}(f)$, hence $\operatorname{ker} \varphi_{*} \cap \operatorname{ker} f_{*}=\{0\}$. Then $\operatorname{dim} \varphi_{*}\left(\operatorname{ker} f_{*}\right)=\operatorname{dim} \operatorname{ker} g_{*} ;$ on the other hand, $\varphi_{*}\left(\operatorname{ker} f_{*}\right) \subseteq \operatorname{ker} g_{*}$, so equality holds.

Observe that $\varphi$ is an isomorphism over the general fibers of $f$ and $g$. Let $F_{1}$ be a general fiber of $f$, so that $\mathcal{N}_{1}\left(F_{1}, X\right)=\operatorname{ker} f_{*}$ because $f$ is quasi-elementary. Then $\varphi\left(F_{1}\right)$ is a general fiber of $g$, and $\mathcal{N}_{1}\left(\varphi\left(F_{1}\right), W\right)=\varphi_{*}\left(\mathcal{N}_{1}\left(F_{1}, X\right)\right)=\varphi_{*}\left(\operatorname{ker} f_{*}\right)=\operatorname{ker} g_{*}$. Thus $g$ is quasi-elementary.

Then $Z$ is factorial with canonical singularities by Lemma 3.10(i). Let us show that $-K_{Y}$. $\operatorname{NE}(\psi) \geqslant 0$. Write $K_{Y}=\psi^{*}\left(K_{Z}\right)+r \operatorname{Exc}(\psi)$ with $r \in \mathbb{Z}$, and let $C_{1} \subset Y$ be a curve contracted by $\psi$. Then $\operatorname{Exc}(\psi) \cdot C_{1}<0$ by Remark 2.4 , and $-K_{Y} \cdot C_{1}=r\left(-\operatorname{Exc}(\psi) \cdot C_{1}\right)$. Hence we have to show that $r \geqslant 0$. Let $h: Y^{\prime} \rightarrow Y$ be a resolution of singularities of $Y$, and consider the composition $\psi \circ h: Y^{\prime} \rightarrow Z$. Call $E^{\prime}$ the proper transform of $\operatorname{Exc}(\psi)$ in $Y^{\prime}$. Then $r$ is the coefficient of $E^{\prime}$ in $K_{Y^{\prime}}-(\psi \circ h)^{*}\left(K_{Z}\right)$, thus $r \geqslant 0$ because $Z$ has canonical singularities.

Assume now that $\operatorname{dim} Y=3$ and set $S:=\operatorname{Exc}(\psi)$.

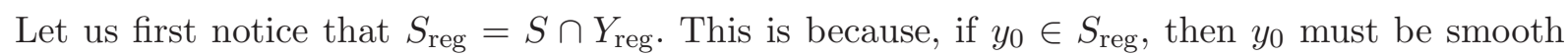
for $Y$ too, because $S$ is a Cartier divisor in $Y$. On the other hand, let $y_{1} \in Y_{\text {reg }}$ and let $h \in \mathcal{O}_{Y, y_{1}}$ be a local equation for $S$ in $y_{1}$. Since $E=f^{*}(S), f^{*}(h)$ is a local equation for $E$ near the fiber over $y_{1}$. Take $x_{1}$ in this fiber. Since $E$ is smooth, the differential $d_{x_{1}}\left(f^{*}(h)\right)$ is non-zero, and since $d_{x_{1}}\left(f^{*}(h)\right)=d_{y_{1}} h \circ d_{x_{1}} f$, the differential $d_{y_{1}} h$ must be non-zero too. Thus $S$ is smooth at $y_{1}$. 


\section{QUASI-ELEMENTARY CONTRACTIONS OF FANO MANIFOLDS}

Recall that $Y$ has rational singularities, in particular it is Cohen-Macaulay (see [Kol86, Corollary 7.4] and [KM98, Theorem 5.10]). Since $S$ is a Cartier divisor in $Y$, it is Cohen-Macaulay too. On the other hand, Lemma 3.10(iv) implies that $Y$ and $S$ have isolated singularities. Then $S$ is normal by Serre's criterion.

By Lemma $3.9 f$ can have at most isolated fibers of dimension $n-2$. Let us show that $f$ is equidimensional over $S$. If $F_{0} \subset E$ is an irreducible component of a fiber of $f$ with $\operatorname{dim} F_{0}=n-2$, then $\varphi\left(F_{0}\right) \subseteq \varphi(E)$ and $\operatorname{dim} \varphi\left(F_{0}\right)=n-2=\operatorname{dim} \varphi(E)$, hence $\varphi\left(F_{0}\right)=\varphi(E)$. This gives

$$
\psi(S)=\psi(f(E))=g(\varphi(E))=g\left(\varphi\left(F_{0}\right)\right)=\psi\left(f\left(F_{0}\right)\right)=p t,
$$

a contradiction.

Now $E$ is smooth, $S$ is normal, and $f_{\mid E}: E \rightarrow S$ has connected fibers. As in the proof of Lemma 3.10(iv) we see that $f_{\mid E}$ is a Mori contraction, and by Lemma 3.10(ii) the surface $S$ is smooth, so that $S \cap \operatorname{Sing}(Y)=\emptyset$.

The general fiber $l$ of $\psi_{\mid S}: S \rightarrow \psi(S)$ is a rational curve, and it is smooth because $S$ is, hence $-K_{S} \cdot l=2$.

Let $\tilde{l}$ be a fiber of $\varphi$ such that $f(\tilde{l})=l$. Then

$$
-1=\tilde{l} \cdot E=\tilde{l} \cdot f^{*}(S)=f_{*}(\tilde{l}) \cdot S,
$$

which gives $l \cdot S=-1$ and $-K_{Y} \cdot l=-K_{S} \cdot l+S \cdot l=1$.

This shows also that $\psi_{\mid S}: S \rightarrow \psi(S)$ is a $\mathbb{P}^{1}$-bundle. Therefore $\psi$ is the blow-up of a smooth curve contained in the smooth locus of $Z$, and $Z$ has the same singularities as $Y$.

Finally, let us suppose that $\operatorname{dim} Y=2$. Then $Y$ and $Z$ are smooth by Lemma 3.10(iii), and $f$ and $g$ are equidimensional. Hence $\psi$ is the blow-up of a point $z_{0} \in Z$. We have

$$
E=f^{-1}(\operatorname{Exc}(\psi))=\varphi^{-1}\left(g^{-1}\left(z_{0}\right)\right),
$$

so the center of $\varphi$ is $\varphi(E)=g^{-1}\left(z_{0}\right)=F_{0}$.

Since $E=f^{*}(\operatorname{Exc}(\psi)), E$ is the (schematic) fiber of $\psi \circ f$ over $z_{0}$, and it is reduced. Thus $g \circ \varphi$ has reduced fiber over $z_{0}$, and the same must hold for $g$. Now $g$ is an equidimensional morphism between smooth varieties, whose fiber $F_{0}$ over $z_{0}$ is reduced and smooth. This implies that $g$ is smooth over $z_{0}$, and hence the normal bundle of $F_{0}$ in $W$ is trivial. Since $E$ is the exceptional divisor of the blow-up of $F_{0}$, we deduce that $E \cong \mathbb{P}^{1} \times F_{0}$, and $f_{\mid E}$ and $\varphi_{\mid E}$ are the two projections.

Suppose that $X$ is Fano and $W$ is not. Then [Wiś91a, Proposition 3.4] says that there exists an extremal ray $\alpha$ of $\operatorname{NE}(X)$, different from $\operatorname{NE}(\varphi)$, such that $\alpha \cdot E<0$. This implies that $\alpha$ is not contained in $\mathrm{NE}(f)$, because $C \cdot E=0$ for every curve $C$ contracted by $f$. However $E \cong \mathbb{P}^{1} \times F_{0}$, so every curve in $E$ is numerically equivalent to a linear combination with coefficients in $\mathbb{Q} \geqslant 0$ of a curve in $\mathrm{NE}(f)$ and a curve in $\mathrm{NE}(\varphi)$. Thus no curve in $E$ can have numerical class in an extremal ray $\alpha$ not contained in $\mathrm{NE}(f) \cup \mathrm{NE}(\varphi)$, and we have a contradiction. This completes the proof of Theorem 3.14.

\subsection{Divisors $D$ with small $\operatorname{dim} \mathcal{N}_{1}(D, X)$}

Let $X$ be a smooth Fano variety of dimension $n$, and $D$ a prime divisor in $X$. If $D$ is simple, e.g. if $\rho_{D}$ is very small, then one can hope to deduce information on $X$ itself. For instance, in [BCW02] the authors classify the possible pairs $(X, D)$ when $D \cong \mathbb{P}^{n-1}$ and $\mathcal{N}_{D / X} \cong \mathcal{O}_{\mathbb{P}^{n-1}}(-1)$. This is equivalent to asking that $X$ is obtained by blowing up a smooth variety in a point.

This classification has been generalized in [Tsu06] to the case $D \cong \mathbb{P}^{n-1}$ and $\mathcal{N}_{D / X} \cong \mathcal{O}_{\mathbb{P}^{n-1}}(-a)$ with $a \in \mathbb{Z}_{\geqslant 1}$. 


\section{Casagrande}

With the same techniques, [Tsu06, Proposition 5] shows that, if $X$ contains a prime divisor $D$ with $\rho_{D}=1$ and $\operatorname{dim} X \geqslant 3$, then $\rho_{X} \leqslant 3$. The proof of [Tsu06, Proposition 5] can be generalized in order to obtain the following result.

Proposition 3.16. Let $X$ be a smooth Fano variety, and let $f: X \rightarrow Y$ be either a quasi-elementary contraction of fiber type or an isomorphism.

Suppose that $\operatorname{dim} Y \geqslant 3$ and that there exists a prime divisor $D \subset Y$ such that $\operatorname{dim} \mathcal{N}_{1}(D, Y)=1$. Then $\rho_{Y} \leqslant 3$.

In the case where $f$ is an isomorphism, this says that if $\operatorname{dim} X \geqslant 3$ and $X$ contains a prime divisor $D$ with $\operatorname{dim} \mathcal{N}_{1}(D, X)=1$, then $\rho_{X} \leqslant 3$. In particular, if $\operatorname{dim} X=n \geqslant 3$ and $X$ has an elementary contraction of type $(n-1,0)$, then $\rho_{X} \leqslant 3$.

Proof. Recall that $Y$ is factorial by Lemma 3.10(i). Moreover $\mathrm{NE}(Y)$ is closed and polyhedral, and every extremal ray can be contracted, by Lemma 2.6.

Let us assume that $\rho_{Y}>1$. There exists at least one extremal ray $\alpha_{1}$ of $\operatorname{NE}(Y)$ such that $\alpha_{1} \cdot D>0$; let $\psi_{1}: Y \rightarrow Z_{1}$ be its contraction. Then $\operatorname{dim} Z_{1}>0$ and $D$ must intersect every non-trivial fiber of $\psi_{1}$.

If $\alpha_{1} \subset \mathcal{N}_{1}(D, Y)$, then every curve in $D$ has numerical class contained in $\alpha_{1}$, hence $\psi_{1}(D)$ is a point. Then $\psi_{1}$ cannot be of fiber type (otherwise $Z_{1}$ is a point), so $\psi_{1}$ is birational and $D \subseteq \operatorname{Exc}\left(\psi_{1}\right)$. This implies that $D \cdot \alpha_{1}<0$ by Remark 2.4, a contradiction.

Hence $\alpha_{1} \not \subset \mathcal{N}_{1}(D, Y)$. Consider a non-trivial fiber $F$ of $\psi_{1}$. Then $F \cap D \neq \emptyset$; on the other hand $\operatorname{dim}(F \cap D)=0$ otherwise we would get a curve in $D$ with numerical class in $\alpha_{1}$. Since $Y$ is factorial, we get

$$
0=\operatorname{dim}(F \cap D) \geqslant \operatorname{dim} F-1
$$

so $F$ has dimension 1.

If $\psi_{1}$ is of fiber type, then

$$
\left(\psi_{1}\right)_{* \mid \mathcal{N}_{1}(D, Y)}: \mathcal{N}_{1}(D, Y) \longrightarrow \mathcal{N}_{1}\left(Z_{1}\right)
$$

is surjective, so $\rho_{Z_{1}}=1$ and $\rho_{Y}=2$.

Assume that $\psi_{1}$ is birational and consider a lifting of $\psi_{1}$ as in $\S 2.5$.

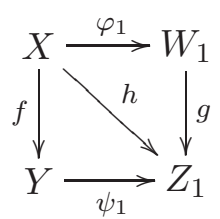

Since $X$ is Fano, $\varphi_{1}$ is a Mori contraction, and Theorem 3.14 applies. Thus $g$ is a quasi-elementary Mori contraction, $W_{1}$ is smooth, $\varphi_{1}$ is a blow-up, $\psi_{1}$ is divisorial, $Z_{1}$ is factorial, and $\operatorname{Exc}\left(\varphi_{1}\right)=$ $f^{-1}\left(\operatorname{Exc}\left(\psi_{1}\right)\right)$.

Since $\psi_{1}$ is finite on $D, D_{2}:=\psi_{1}(D)$ is a Cartier divisor in $Z_{1}$. Moreover

$$
\left(\psi_{1}\right)_{* \mid \mathcal{N}_{1}(D, Y)}: \mathcal{N}_{1}(D, Y) \longrightarrow \mathcal{N}_{1}\left(D_{2}, Z_{1}\right)
$$

is surjective, hence $\operatorname{dim} \mathcal{N}_{1}\left(D_{2}, Z_{1}\right)=1$. Recall also that $D$ intersects every non-trivial fiber of $\psi_{1}$, hence $D_{2} \supset \psi_{1}\left(\operatorname{Exc}\left(\psi_{1}\right)\right)$.

Again by Lemma 2.6, $\mathrm{NE}\left(Z_{1}\right)$ is closed and polyhedral, and every extremal ray can be contracted.

Thus consider an extremal ray $\alpha_{2}$ of $\mathrm{NE}\left(Z_{1}\right)$ such that $\alpha_{2} \cdot D_{2}>0$, and let $\psi_{2}: Z_{1} \rightarrow Z_{2}$ be the associated contraction.

As before, we see that if $\psi_{2}$ is of fiber type then $\rho_{Z_{1}} \leqslant 2$ and $\rho_{Y} \leqslant 3$. 


\section{QUASI-ELEMENTARY CONTRACTIONS OF FANO MANIFOLDS}

If $\psi_{2}$ is birational, then $\alpha_{2} \not \subset \mathcal{N}_{1}\left(D_{2}, Z_{1}\right)$ and again every non-trivial fiber of $\psi_{2}$ has dimension 1 . We show that this case leads to a contradiction.

Let $\widehat{\alpha}_{2}$ be the unique extremal face of $\mathrm{NE}(X)$ such that $\widehat{\alpha}_{2}$ contains $\mathrm{NE}(h)$ and $h_{*}\left(\widehat{\alpha}_{2}\right)=\alpha_{2}$ (see $\S 2.5)$. Notice that $\mathrm{NE}\left(\varphi_{1}\right)$ is an extremal ray of $\mathrm{NE}(h)$.

Let us make an easy remark on convex polyhedral cones. If $\sigma \subset \mathbb{R}^{d}$ is a convex polyhedral cone, $\gamma$ is a proper face of $\sigma$, and $\rho$ is an extremal ray of $\gamma$, then there exists an extremal ray $\rho_{2}$ of $\sigma$, not contained in $\gamma$, and such that $\rho+\rho_{2}$ is a face of $\sigma$. The reader who is not familiar with convex geometry may easily prove this by induction on $\operatorname{dim} \sigma$.

Applied to our situation, this says that there is an extremal ray $\widetilde{\beta}$ of $\widehat{\alpha}_{2}$ such that $\widetilde{\beta} \not \subset \operatorname{NE}(h)$ and $\widetilde{\beta}+\mathrm{NE}\left(\varphi_{1}\right)$ is a face of $\widehat{\alpha}_{2}$.

Then $\beta=\left(\varphi_{1}\right)_{*}(\widetilde{\beta})$ is an extremal ray of $\mathrm{NE}\left(W_{1}\right)$, whose contraction $\varphi_{2}: W_{1} \rightarrow W_{2}$ yields the following commutative diagram.

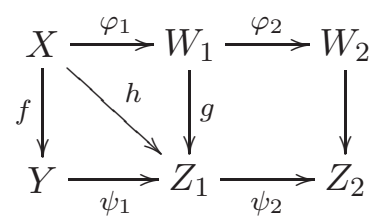

Observe that $\operatorname{NE}\left(\psi_{2} \circ h\right)=\widehat{\alpha}_{2}$ and $\operatorname{NE}\left(\varphi_{2} \circ \varphi_{1}\right)=\widetilde{\beta}+\mathrm{NE}\left(\varphi_{1}\right) \subseteq \mathrm{NE}\left(\psi_{2} \circ h\right)$, so the morphism $W_{2} \rightarrow Z_{2}$ exists by rigidity (see $\S 2.5$ ). Since $\psi_{2}$ is birational and has fibers of dimension at most 1 , it is easy to see that the same holds for $\varphi_{2}$.

Suppose that there exists a non-trivial fiber $C$ of $\varphi_{2}$ contained in $\varphi_{1}\left(\operatorname{Exc}\left(\varphi_{1}\right)\right)$. Let $\widetilde{C}$ be an irreducible curve in $\operatorname{Exc}\left(\varphi_{1}\right)$ such that $\varphi_{1}(\widetilde{C})=C$. Then $f(\widetilde{C}) \subset f\left(\operatorname{Exc}\left(\varphi_{1}\right)\right)=\operatorname{Exc}\left(\psi_{1}\right)$ and $h(\widetilde{C}) \subseteq \psi_{1}\left(\operatorname{Exc}\left(\psi_{1}\right)\right) \subset D_{2}$.

On the other hand, since $\varphi_{2}(C)$ is a point, $\psi_{2}(h(\widetilde{C}))$ must also be a point, namely the numerical class of $h(\widetilde{C})$ must be in $\alpha_{2}=\mathrm{NE}\left(\psi_{2}\right)$. But this contradicts $\alpha_{2} \not \subset \mathcal{N}_{1}\left(D_{2}, Z_{1}\right)$.

Therefore if $C$ is a non-trivial fiber of $\varphi_{2}$, we have $C \nsubseteq \varphi_{1}\left(\operatorname{Exc}\left(\varphi_{1}\right)\right)$, and this implies that $-K_{W_{1}} \cdot C>0$.

Thus $\varphi_{2}$ is a Mori contraction, and Theorem 3.14 yields that $W_{2}$ is smooth, $\varphi_{2}$ is the blowup of a smooth subvariety of codimension 2 in $W_{2}$, and $\operatorname{Exc}\left(\varphi_{2}\right)=g^{-1}\left(\operatorname{Exc}\left(\psi_{2}\right)\right)$. In particular, $-K_{W_{1}} \cdot C=1$.

Now using Remark 2.3 we see that any non-trivial fiber $C$ of $\varphi_{2}$ cannot intersect $\varphi_{1}\left(\operatorname{Exc}\left(\varphi_{1}\right)\right)$, hence

$$
\operatorname{Exc}\left(\varphi_{2}\right) \cap \varphi_{1}\left(\operatorname{Exc}\left(\varphi_{1}\right)\right)=\emptyset .
$$

Recall that $\operatorname{Exc}\left(\varphi_{1}\right)=f^{-1}\left(\operatorname{Exc}\left(\psi_{1}\right)\right)$, which gives $\varphi_{1}\left(\operatorname{Exc}\left(\varphi_{1}\right)\right)=g^{-1}\left(\psi_{1}\left(\operatorname{Exc}\left(\psi_{1}\right)\right)\right)$. Therefore

$$
\emptyset=g^{-1}\left(\operatorname{Exc}\left(\psi_{2}\right)\right) \cap g^{-1}\left(\psi_{1}\left(\operatorname{Exc}\left(\psi_{1}\right)\right)\right)=g^{-1}\left(\operatorname{Exc}\left(\psi_{2}\right) \cap \psi_{1}\left(\operatorname{Exc}\left(\psi_{1}\right)\right)\right),
$$

namely $\operatorname{Exc}\left(\psi_{2}\right) \cap \psi_{1}\left(\operatorname{Exc}\left(\psi_{1}\right)\right)=\emptyset$.

Notice that $\operatorname{dim} \psi_{1}\left(\operatorname{Exc}\left(\psi_{1}\right)\right)=\operatorname{dim} Y-2 \geqslant 1$, so there exists a curve $C_{2} \subseteq \psi_{1}\left(\operatorname{Exc}\left(\psi_{1}\right)\right)$, and $C_{2} \cdot \operatorname{Exc}\left(\psi_{2}\right)=0$.

On the other hand $\psi_{1}\left(\operatorname{Exc}\left(\psi_{1}\right)\right) \subset D_{2}$ and $\operatorname{dim} \mathcal{N}_{1}\left(D_{2}, Z_{1}\right)=1$. This implies that $C_{2}^{\prime} \cdot \operatorname{Exc}\left(\psi_{2}\right)=0$ for every curve $C_{2}^{\prime} \subset D_{2}$. But this is impossible, because $D_{2}$ and $\operatorname{Exc}\left(\psi_{2}\right)$ are distinct prime divisors with non-empty intersection. This completes the proof of Proposition 3.16.

Remark 3.17. More precisely we have shown that in the hypotheses of Proposition 3.16, either $\rho_{Y}=1$, or one of the following occurs: 


\section{Casagrande}

(a) $\rho_{Y}=2$ and $Y$ has an elementary contraction of fiber type with one-dimensional fibers; or

(b) $\rho_{Y}=3$ and $Y$ has a divisorial elementary contraction such that every fiber has dimension at most 1 .

\section{Existence of contractions after [BCD07]}

Let $X$ be any normal projective variety. Consider an irreducible closed subset $V$ of $\operatorname{Chow}(X)$ such that:

(a) for $v \in V$ general, the corresponding cycle $C_{v} \subset X$ is an irreducible, reduced, and connected rational curve; and

(b) every point of $X$ is contained in $C_{v}$ for some $v \in V$.

We call $V$ a covering family of rational curves in $X$. Such a family induces an equivalence relation on $X$ (as a set), called $V$-equivalence, as follows. Two points $x, y \in X$ are $V$-equivalent if there exist $v_{1}, \ldots, v_{m} \in V$ such that $C_{v_{1}} \cup \cdots \cup C_{v_{m}}$ is connected and contains $x$ and $y$. This notion was originally introduced by Campana [Cam81] and is by now well known; see [Kol96, § IV.4], [Deb01, $\S 5]$, and [Cam04]. We refer specifically to [BCD07] for the set-up and for precise references.

In particular it is known that there exist an open subset $X_{0}$ that is closed under $V$-equivalence, a normal quasi-projective variety $Y_{0}$, and a proper, equidimensional morphism $q: X_{0} \rightarrow Y_{0}$, such that every fiber of $q$ is a $V$-equivalence class. In general, there are no morphisms defined on the whole $X$ that extend $q$.

If $f: X \rightarrow Y$ is a Mori contraction of fiber type, then one can find a family $V$ as above such that $q=f_{\mid X_{0}}$ (see the proof of Theorem 4.1). Using the properties of this family, we can apply the results of [BCD07] to deduce the following result.

Theorem 4.1. Let $X$ be a smooth variety of dimension $n$ and consider two Mori contractions

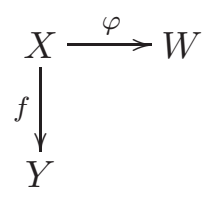

where $f$ is elementary of fiber type and $\mathrm{NE}(f) \cap \mathrm{NE}(\varphi)=\{0\}$. Let $k_{f}$ and $k_{\varphi}$ be the dimensions of the general fibers of $f$ and $\varphi_{\mid \operatorname{Exc}(\varphi)}$ respectively. Assume that we are in one of the following situations:

(i) $\varphi$ is quasi-elementary of fiber type, and $k_{f}+k_{\varphi} \geqslant n-3$;

(ii) $\varphi$ is elementary and divisorial, and $\operatorname{dim} Y \leqslant 3$; or

(iii) $\varphi$ is elementary and divisorial, $f(\operatorname{Exc}(\varphi))=Y$, and $k_{\varphi} \geqslant \operatorname{dim} Y-3$.

Then there exists a commutative diagram

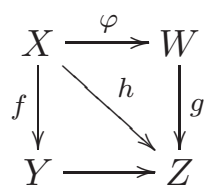

where $g: W \rightarrow Z$ is an elementary Mori contraction and $\operatorname{dim} Z \leqslant 3$.

Let us point out that we do not know whether the hypotheses on $k_{f}+k_{\varphi}, \operatorname{dim} Y$, and $k_{\varphi}$ respectively are really necessary for the statement to hold.

Proof. In the first part of the proof we will just assume that $f$ is quasi-elementary and that $\operatorname{NE}(f) \cap$ $\mathrm{NE}(\varphi)=\{0\}$. 


\section{QUASI-ELEMENTARY CONTRACTIONS OF FANO MANIFOLDS}

We first construct a suitable covering family $V$ of rational curves in $X$, such that $f$ is the quotient for $V$-equivalence over an open subset of $X$.

Let $F$ be a general fiber of $f$, then $F$ is a smooth Fano variety of dimension $k_{f}$. In particular $F$ is rationally connected, and there exists a smooth rational curve $C_{0} \subset F$ that is very free, namely with ample normal bundle in $F$ (see [Deb01, $\S \S 4.3$ and 5.6]):

$$
\mathcal{N}_{C_{0} / F} \cong \bigoplus_{i} \mathcal{O}_{\mathbb{P}^{1}}\left(a_{i}\right), \quad a_{i} \in \mathbb{Z}_{>0} \text { for every } i .
$$

Since $\mathcal{N}_{F / X}$ is trivial, we have

$$
\mathcal{N}_{C_{0} / X} \cong \mathcal{O}_{\mathbb{P}^{1}}^{\oplus\left(n-k_{f}\right)} \oplus \bigoplus_{i} \mathcal{O}_{\mathbb{P}^{1}}\left(a_{i}\right),
$$

hence $C_{0}$ is a free curve in $X$. This means that the deformations of $C_{0}$ cover the whole $X$ : by [Deb01, Proposition 4.8] there exists a covering family $V$ of rational curves in $X$ such that $C_{0}=C_{v_{0}}$ for some $v_{0} \in V$.

Clearly all curves parametrized by $V$ are numerically equivalent in $X$, and their numerical class is contained in $\mathrm{NE}(f)$, because $f\left(C_{0}\right)$ is a point. Since $\mathrm{NE}(f)$ is a face of $\mathrm{NE}(X)$, we deduce that every irreducible component of every curve parametrized by $V$ is contained in some fiber of $f$. This implies that every $V$-equivalence class is contained in some fiber of $f$.

Consider the quotient for $V$-equivalence $q: X_{0} \rightarrow Y_{0}$; let $F_{0}$ be a general fiber, and $C_{v} \subset F_{0}$ a general curve of the family. Then $C_{v} \cong \mathbb{P}^{1}$ and we have

$$
\mathcal{N}_{C_{v} / X} \cong \mathcal{O}_{\mathbb{P}^{1}}^{\oplus\left(n-\operatorname{dim} F_{0}\right)} \oplus \mathcal{N}_{C_{v} / F_{0}} .
$$

On the other hand, by [Kol96, II.3.9.2] the number of trivial summands in $\mathcal{N}_{C_{v} / X}$ is at most the number of trivial summands in $\mathcal{N}_{C_{0} / X}$, so that $n-\operatorname{dim} F_{0} \leqslant n-k_{f}$ and $\operatorname{dim} F_{0} \geqslant k_{f}$.

This implies that $\operatorname{dim} F_{0}=k_{f}$ and hence all fibers of $f$ of dimension $k_{f}$ are $V$-equivalence classes.

We observe that $V$ induces in a natural way a covering family of rational curves on $W$. First of all, the hypothesis $\mathrm{NE}(f) \cap \mathrm{NE}(\varphi)=\{0\}$ implies that $\varphi$ does not contract any irreducible component of any curve of $V$.

Consider now the incidence diagram associated to $V$, shown below.

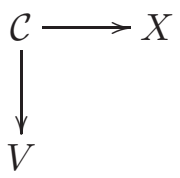

We proceed as in [BCD07, proof of Lemma 2]. Let $\widetilde{\mathcal{C}}$ be the normalization of $\mathcal{C}$ and $\widetilde{\mathcal{C}} \rightarrow \widetilde{V}$ be the Stein factorization of the composite map $\widetilde{\mathcal{C}} \rightarrow \mathcal{C} \rightarrow V$. Then $\widetilde{V}$ is normal, the general fiber of $\widetilde{\mathcal{C}} \rightarrow \widetilde{V}$ is $\mathbb{P}^{1}$, and the composite map

$$
\widetilde{\mathcal{C}} \longrightarrow \mathcal{C} \longrightarrow X \stackrel{\varphi}{\longrightarrow} W
$$

yields a family of 1-cycles in $W$, thus a morphism $\widetilde{V} \rightarrow \operatorname{Chow}(W)$. We call $V^{\prime}$ the image of this morphism. By the construction, for every irreducible component $C$ of a curve parametrized by $V$, the image $\varphi(C)$ is a component of some curve parametrized by $V^{\prime}$, and conversely. In particular, if $x, y \in X$ are $V$-equivalent, then $\varphi(x), \varphi(y) \in W$ are $V^{\prime}$-equivalent.

Claim. Let $T \subseteq W$ be a general $V^{\prime}$-equivalence class. Then $\operatorname{codim} T \leqslant n-k_{f}$, and $\operatorname{codim} T \leqslant$ $n-\left(k_{f}+k_{\varphi}\right)$ if $f(\operatorname{Exc}(\varphi))=Y$.

Observe that the second case always holds if $\varphi$ is of fiber type. 


\section{Casagrande}

Proof of the claim. The inverse image $\varphi^{-1}(T)$ is closed for $V$-equivalence. Let $F$ be a general $V$ equivalence class contained in $\varphi^{-1}(T)$, so that $F$ is a fiber of $f$. Since $\varphi$ is finite on $F$ and $\varphi(F) \subseteq T$, we have $\operatorname{dim} T \geqslant \operatorname{dim} F=k_{f}$, while $\operatorname{dim} W \leqslant n$, which gives the first statement.

If $\varphi$ is of fiber type, then $\operatorname{dim} W=n-k_{\varphi}$, so the same $\operatorname{argument}$ gives $\operatorname{codim} T \leqslant n-\left(k_{f}+k_{\varphi}\right)$.

Let us assume that $\varphi$ is birational and that $\operatorname{Exc}(\varphi)$ dominates $Y$ via $f$. Then $F \cap \operatorname{Exc}(\varphi) \neq \emptyset$, so $T \cap \varphi(\operatorname{Exc}(\varphi)) \neq \emptyset$, and since $T$ is general, every $V^{\prime}$-equivalence class intersects $\varphi(\operatorname{Exc}(\varphi))$. This means that $\varphi^{-1}(T)$ contains a general fiber $N$ of $\varphi_{\mid \operatorname{Exc}(\varphi)}$. Let $N_{0}$ be an irreducible component of $N$ with $\operatorname{dim} N_{0}=k_{\varphi}$.

There exists a non-empty open subset $U_{0}$ of $f\left(N_{0}\right)$ such that every fiber of $f$ over $U_{0}$ is an irreducible $V$-equivalence class. Then $f^{-1}\left(U_{0}\right)$ is irreducible of dimension $k_{f}+k_{\varphi}$, and it must be contained in $\varphi^{-1}(T)$ because $\varphi^{-1}(T)$ is closed for $V$-equivalence. Moreover $\varphi_{\mid f^{-1}\left(U_{0}\right)}$ is birational, so $\operatorname{dim} T \geqslant k_{f}+k_{\varphi}$. This completes the proof of the claim.

Consider now the linear subspaces $H_{V}$ of $\mathcal{N}_{1}(X)$ and $H_{V^{\prime}}$ of $\mathcal{N}_{1}(W)$ generated by the numerical classes of all irreducible components of all curves in $V$ and $V^{\prime}$ respectively. Then we have $\varphi_{*}\left(H_{V}\right)=$ $H_{V^{\prime}}$; and moreover $\mathcal{N}_{1}(F, X) \subseteq H_{V}$ and $\mathcal{N}_{1}(T, W) \subseteq H_{V^{\prime}}$ by [Kol96, Proposition IV.3.13.3] (see also [BCD07, Remark 1]).

Since $f$ is quasi-elementary, we get $\operatorname{ker} f_{*}=\mathcal{N}_{1}(F, X) \subseteq H_{V} \subseteq \operatorname{ker} f_{*}$, hence $\mathcal{N}_{1}(F, X)=H_{V}=$ ker $f_{*}$. Then we have

$$
\mathcal{N}_{1}(T, W) \subseteq H_{V^{\prime}}=\varphi_{*}\left(H_{V}\right)=\varphi_{*}\left(\mathcal{N}_{1}(F, X)\right)=\mathcal{N}_{1}(\varphi(F), W) \subseteq \mathcal{N}_{1}(T, W),
$$

hence

$$
\mathcal{N}_{1}(T, W)=H_{V^{\prime}}=\varphi_{*}\left(\mathcal{N}_{1}(F, X)\right)=\varphi_{*}\left(\operatorname{ker} f_{*}\right) .
$$

We observe that $\varphi$ is either elementary and divisorial, or quasi-elementary of fiber type. Then in any case $W$ is $\mathbb{Q}$-factorial and has canonical singularities, by Lemma 3.10 and [Deb01, Proposition 7.44].

Let us assume now that $f$ is an elementary contraction. Then $\operatorname{dim} H_{V}=\operatorname{dim} H_{V^{\prime}}=1$, and this means that $V$ and $V^{\prime}$ are quasi-unsplit in the sense of [BCD07]. Moreover, using the claim we see that in any case the general $V^{\prime}$-equivalence class has codimension at most 3 . Hence [BCD07, Theorem 2] yields the existence of an elementary Mori contraction $g: W \rightarrow Z$ such that every fiber of $g$ is a $V^{\prime}$-equivalence class. Then $\operatorname{dim} Z \leqslant 3$, and by rigidity (see for instance [Deb01, Lemma 1.15]) there exists a morphism $Y \rightarrow Z$ as in the statement. The completes the proof of Theorem 4.1.

We observe that, with a slight modification of the argument, we can prove a different version of the previous theorem. Namely we can allow $f$ to be quasi-elementary instead of elementary, if we impose a stronger condition on $k_{f}$ and $k_{\varphi}$.

Theorem 4.4. Let $X$ be a smooth variety of dimension $n$ and consider two Mori contractions $f: X \rightarrow Y$ and $\varphi: X \rightarrow W$ such that $\mathrm{NE}(f) \cap \mathrm{NE}(\varphi)=\{0\}$ and $f$ is quasi-elementary of fiber type. Let $k_{f}$ and $k_{\varphi}$ be the dimensions of the general fibers of $f$ and $\varphi_{\mid \operatorname{Exc}(\varphi)}$ respectively. Assume that we are in one of the following situations:

(i) $\varphi$ is quasi-elementary and $k_{f}+k_{\varphi} \geqslant n-2$;

(ii) $\varphi$ is elementary and divisorial, and $\operatorname{dim} Y \leqslant 2$; or

(iii) $\varphi$ is elementary and divisorial, $f(\operatorname{Exc}(\varphi))=Y$, and $k_{\varphi} \geqslant \operatorname{dim} Y-2$.

Then there exists a commutative diagram as (4.2) where $h: X \rightarrow Z$ is a contraction, $\operatorname{dim} Z \leqslant 2$, and $\rho_{X}-\rho_{Z} \leqslant\left(\rho_{X}-\rho_{Y}\right)+\left(\rho_{X}-\rho_{W}\right)$ (equality holds except possibly in (i)). 


\section{QUASI-ELEMENTARY CONTRACTIONS OF FANO MANIFOLDS}

Proof. We perform the same construction as in the previous proof, so that $W$ is a normal and $\mathbb{Q}$-factorial projective variety, and $V^{\prime}$ is a covering family of rational curves in $W$. Moreover by the claim and the assumptions, we see that now the general $V^{\prime}$-equivalence class has codimension at most 2 in $W$. On the other hand, $V^{\prime}$ is not quasi-unsplit if $f$ is not elementary. However (4.3) implies the following property.

Let $D$ be a Weil divisor in $W$ whose support is disjoint from the general $V^{\prime}$-equivalence class. Then $D \cdot C=0$ for every irreducible component of every curve in $V^{\prime}$.

We claim that we can apply [BCD07, Proposition 1] to the family $V^{\prime}$ on $W$, even if $V^{\prime}$ is not quasi-unsplit. Indeed the quasi-unsplit assumption is used in the proof of this proposition uniquely to deduce the property above. One can think that the property above generalizes the quasi-unsplit property, in the same way as quasi-elementary contractions generalize elementary contractions of fiber type.

Now as in the proof of [BCD07, Theorem 1], we get the existence of a normal projective variety $Z$ and a surjective morphism $g: W \rightarrow Z$ such that every fiber of $g$ is a $V^{\prime}$-equivalence class (even if $g$ is not necessarily a Mori contraction).

As before this implies the existence of the diagram (4.2) by rigidity, and $h:=g \circ \varphi$ is a contraction. Moreover, it is clear that $\operatorname{ker} g_{*}=H_{V^{\prime}}$ and

$$
\operatorname{ker} h_{*}=\left(\varphi_{*}\right)^{-1}\left(H_{V^{\prime}}\right)=\left(\varphi_{*}\right)^{-1}\left(\varphi_{*}\left(\operatorname{ker} f_{*}\right)\right)=\operatorname{ker} f_{*}+\operatorname{ker} \varphi_{*} .
$$

Observe that the hypothesis $\operatorname{NE}(f) \cap \operatorname{NE}(\varphi)=\{0\}$ does not imply that $\operatorname{ker} f_{*} \cap \operatorname{ker} \varphi_{*}=\{0\}$, unless we know that one of $f$ or $\varphi$ is elementary, as in (ii) or (iii). Thus $\operatorname{dim} \operatorname{ker} h_{*} \leqslant \operatorname{dim} \operatorname{ker} f_{*}+\operatorname{dim} \operatorname{ker} \varphi_{*}$, and equality holds except possibly in (i).

Remark 4.5. In both Theorems 4.1 and 4.4, we have in fact proved that $\operatorname{dim} Z \leqslant \operatorname{dim} Y$ in case (ii), and $\operatorname{dim} Z \leqslant n-\left(k_{f}+k_{\varphi}\right)=\operatorname{dim} Y-k_{\varphi}$ in cases (i) and (iii). Thus the contraction $Y \rightarrow Z$ is of fiber type in case (i), and elementary of fiber type in case (iii).

\section{Fano manifolds with a quasi-elementary contraction onto a surface}

In this section we show Theorem 1.1(i). So let us consider a smooth Fano variety $X$ of dimension $n \geqslant 3$, and a quasi-elementary contraction $f: X \rightarrow Y$ onto a surface. We know by Lemma 3.10(iii) that $f$ is equidimensional and $Y$ is smooth. Moreover, $Y$ is rational, for instance because $X$ is rationally connected.

Remark 5.1. Let $\psi: Y \rightarrow Z$ be a birational elementary contraction. Then $Z$ is smooth, $\psi$ is the blow-up of a point $z_{0} \in Z$, and there exists a diagram

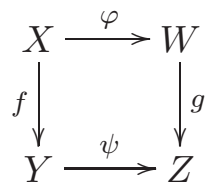

where $W$ is a smooth Fano variety, $g$ is a quasi-elementary contraction with smooth fiber $F_{0}$ over $z_{0}, \varphi$ is the blow-up of $F_{0}, \operatorname{Exc}(\varphi) \cong \mathbb{P}^{1} \times F_{0}$, and $f_{\mid \operatorname{Exc}(\varphi)}, \varphi_{\mid \operatorname{Exc}(\varphi)}$ are the two projections.

In fact $\psi$ has a lifting as explained in $\S 2.5$, which gives the diagram above. Since $X$ is Fano, $\varphi$ is a Mori contraction. Then Theorem $3.14(\mathrm{v})$ gives the statement.

Notice moreover that the lifting $\varphi$ is uniquely determined by $\psi$, because $\operatorname{Exc}(\varphi)=f^{-1}(\operatorname{Exc}(\psi)) \cong$ $\mathbb{P}^{1} \times F_{0}$ and $\mathrm{NE}(\varphi)$ is determined by the curves $\mathbb{P}^{1} \times\{p t\}$ in $X$. As described in $\S 2.5$, every choice of an extremal ray $\widetilde{\alpha}$ in $\mathrm{NE}(\psi \circ f)$ such that $\widetilde{\alpha} \nsubseteq \mathrm{NE}(f)$, gives rise to a lifting of $\psi$. We deduce that such an extremal ray $\widetilde{\alpha}=\mathrm{NE}(\varphi)$ is unique, and that $\mathrm{NE}(\psi \circ f)=\widetilde{\alpha}+\mathrm{NE}(f)$. 


\section{Casagrande}

Proof of Theorem 1.1(i). If $\rho_{Y}=1$ then $Y \cong \mathbb{P}^{2}$, so we can assume that $\rho_{Y}>1$. In order to show that $Y$ is Del Pezzo, it is enough to show that any elementary contraction of $Y$ is a Mori contraction, see Remark 3.11.

Let us fix such a contraction $\psi: Y \rightarrow Z$. If $\psi$ is birational, then Remark 5.1 says that $Z$ is smooth and $\psi$ is a blow-up, hence a Mori contraction.

Let us consider the case where $\psi$ is not birational, so that $\operatorname{dim} Z \leqslant 1$. Since we have assumed that $\rho_{Y}>1$, we have $\operatorname{dim} Z=1$, and $Y$ is a smooth rational surface with $\rho_{Y}=2$. Thus $Y$ is a Hirzebruch surface and $Z \cong \mathbb{P}^{1}$. If we consider the other elementary contraction of $Y$, we find two possibilities: either it is again a contraction over $\mathbb{P}^{1}$ and $Y \cong \mathbb{P}^{1} \times \mathbb{P}^{1}$, or it is birational. In this case, by Remark 5.1 it must be a smooth blow-up, so $Y \cong \mathbb{F}_{1}$.

Hence $Y$ is a Del Pezzo surface, and we have the first part of the statement.

Let us assume that $\rho_{Y} \geqslant 3$ and prove the second part of the statement. Since $Y$ is Del Pezzo, there is a morphism $Y \rightarrow \mathbb{P}^{2}$ that is a blow-up of $\rho_{Y}-1$ distinct points $p_{i}$. Thus we can apply Remark 5.1 to each blow-up and get a smooth Fano variety $X_{0}$ with a quasi-elementary contraction $f_{0}: X_{0} \rightarrow \mathbb{P}^{2}$, such that $X$ is obtained from $X_{0}$ by blowing up the fibers of $f_{0}$ over $p_{i}$.

Therefore it is enough to prove the statement in the case $\rho_{Y}=3$, where $Y$ is the blow-up of $\mathbb{P}^{2}$ in two points $p_{1}$ and $p_{2}$. Call $C_{1}$ and $C_{2}$ the two corresponding exceptional curves, and $C_{3}$ the proper transform in $Y$ of the line through $p_{1}$ and $p_{2}$ in $\mathbb{P}^{2}$ (see diagram below).

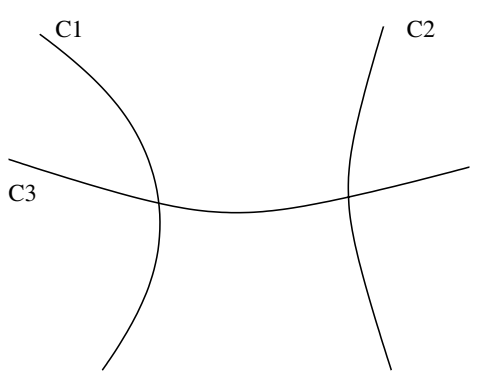

These are all the $(-1)$-curves in $Y$. Call $\alpha_{i}$ the extremal ray of $\mathrm{NE}(Y)$ containing the numerical class of $C_{i}$; then $\operatorname{NE}(Y)=\alpha_{1}+\alpha_{2}+\alpha_{3}$ is simplicial.

Let us make a preliminary remark on the cone $\mathrm{NE}(X)$. The surface $Y$ has three elementary contractions, which are blow-ups with exceptional curves $C_{1}, C_{2}$, and $C_{3}$. Recall from Remark 5.1 that the lifting of each of these blow-ups is unique. This means that for every $i=1,2,3$ there is a unique extremal ray $\widetilde{\alpha}_{i}$ of $\mathrm{NE}(X)$ such that $\widetilde{\alpha}_{i}+\mathrm{NE}(f)$ is a face of $\mathrm{NE}(X)$ and $f_{*}\left(\widetilde{\alpha}_{i}\right)=\alpha_{i}$.

Let us show that $X$ has no small elementary contraction $\xi: X \rightarrow V$ such that $\mathrm{NE}(\xi) \nsubseteq \mathrm{NE}(f)$. By contradiction, let $\xi: X \rightarrow V$ be such a contraction. Since $f$ is finite on fibers of $\xi$, these fibers have dimension at most 2. If $F_{0}$ is an irreducible component of a fiber of $\xi$ with $\operatorname{dim} F_{0}=2$, then $f\left(F_{0}\right)=Y$, so

$$
\left(f_{*}\right)_{\mid \mathcal{N}_{1}\left(F_{0}, X\right)}: \mathcal{N}_{1}\left(F_{0}, X\right) \rightarrow \mathcal{N}_{1}(Y)
$$

is surjective. This is impossible because $\operatorname{dim} \mathcal{N}_{1}\left(F_{0}, X\right)=1$ while $\rho_{Y}=3$.

On the other hand, a small contraction cannot have only one-dimensional fibers; see [Wiś91a, Corollary on p. 145]. Thus we have a contradiction.

Consider now an extremal ray $\beta$ of $\mathrm{NE}(X)$ that is not contained in $\mathrm{NE}(f)$. Since the contraction of $\beta$ is not small, Theorem 4.4(ii) implies that there is a face $\mathrm{NE}(h)$ ( $h$ as in $(4.2)$ ) of $\mathrm{NE}(X)$, of dimension $1+\operatorname{dim} \mathrm{NE}(f)$, containing both $\beta$ and $\mathrm{NE}(f)$. Then $f_{*}(\beta)$ is an extremal ray of $\mathrm{NE}(Y)$, say $\alpha_{1}$. This implies that $\beta=\widetilde{\alpha}_{1}$. 


\section{QUASI-ELEMENTARY CONTRACTIONS OF FANO MANIFOLDS}

Summing up, we have shown that $\widetilde{\alpha}_{1}, \widetilde{\alpha}_{2}$, and $\widetilde{\alpha}_{3}$ are the only extremal rays of $\mathrm{NE}(X)$ outside $\mathrm{NE}(f)$.

Let us show that $X$ is a product. Applying Remark 5.1 we get the sequence

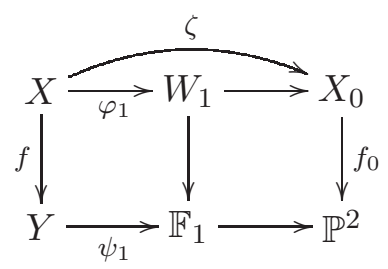

where $\psi_{1}: Y \rightarrow \mathbb{F}_{1}$ is the contraction of $C_{1}$ and $\mathbb{F}_{1} \rightarrow \mathbb{P}^{2}$ is the contraction of (the image of) $C_{2}$; hence $\zeta: X \rightarrow X_{0}$ is the contraction of $\widetilde{\alpha}_{1}+\widetilde{\alpha}_{2}$.

Since $\operatorname{dim} X_{0} \geqslant 3, \mathrm{NE}\left(f_{0}\right)$ is a proper face of $\mathrm{NE}\left(X_{0}\right)$, and there is at least one extremal ray $\beta$ of $\mathrm{NE}\left(X_{0}\right)$ outside $\mathrm{NE}\left(f_{0}\right)$. As explained in $\S 2.5, \beta$ must be the image via $\zeta_{*}$ of some extremal ray $\widetilde{\beta}$ of $\mathrm{NE}(X)$ not lying in $\mathrm{NE}(\zeta)=\widetilde{\alpha}_{1}+\widetilde{\alpha}_{2}$. Since $\zeta_{*}(\mathrm{NE}(f))=\mathrm{NE}\left(f_{0}\right)$ and $\beta \nsubseteq \mathrm{NE}\left(f_{0}\right)$, it must be $\widetilde{\beta} \nsubseteq \mathrm{NE}(f)$. Thus the only possibility is $\widetilde{\beta}=\widetilde{\alpha}_{3}$ and $\beta=\zeta_{*}\left(\widetilde{\alpha}_{3}\right)$.

Let

$$
h: X_{0} \longrightarrow W_{0}
$$

be the contraction of $\beta$. Observe that $\operatorname{dim} W_{0} \geqslant n-2$, because $h$ is finite on fibers of $f_{0}$. Our goal is to show that $X_{0} \cong \mathbb{P}^{2} \times W_{0}$ and $f_{0}$ is the projection, which implies the statement. The first step is to show that $\operatorname{dim} W_{0}=n-2$.

For $i=1,2,3$ let $\varphi_{i}: X \rightarrow W_{i}$ be the smooth blow-up obtained by contracting $\widetilde{\alpha}_{i}$. Let $E_{i} \subset X$ be the exceptional divisor of $\varphi_{i}, F$ the fiber of $f$ over $C_{1} \cap C_{3}$, and $F^{\prime}$ the one over $C_{2} \cap C_{3}$. By Remark 5.1 we have $E_{3} \cong \mathbb{P}^{1} \times F \cong \mathbb{P}^{1} \times F^{\prime}$, so $F \cong F^{\prime}$; let us call it $F$. Thus $E_{i} \cong \mathbb{P}^{1} \times F$, $E_{1} \cap E_{2}=\emptyset$, while $E_{1} \cap E_{3}$ and $E_{2} \cap E_{3}$ are fibers of $f$.

Let $\widetilde{C}_{i} \subset E_{i}$ be a curve corresponding to $\mathbb{P}^{1} \times\{*\}$. Then $\left[\widetilde{C}_{i}\right] \in \widetilde{\alpha}_{i}, \widetilde{C}_{i} \cdot E_{i}=-1, f_{*}\left(\widetilde{C}_{i}\right)=C_{i}$, and $f^{*}\left(C_{i}\right)=E_{i}$. This gives

$$
\widetilde{C}_{1} \cdot E_{3}=\widetilde{C}_{3} \cdot E_{1}=\widetilde{C}_{2} \cdot E_{3}=\widetilde{C}_{3} \cdot E_{2}=1 \quad \text { while } \quad \widetilde{C}_{1} \cdot E_{2}=\widetilde{C}_{2} \cdot E_{1}=0 .
$$

Finally set $E_{3}^{\prime}:=\zeta\left(E_{3}\right)$ and $C_{3}^{\prime}:=\zeta_{*}\left(\widetilde{C}_{3}\right)$, so that $\left[C_{3}^{\prime}\right] \in \beta$.

Observe that $E_{3}^{\prime}$ is a divisor in $X_{0}$ and there are $m_{1}, m_{2} \in \mathbb{Z}_{\geqslant 0}$ such that

$$
\zeta^{*}\left(E_{3}^{\prime}\right)=E_{3}+m_{1} E_{1}+m_{2} E_{2} .
$$

Then

$$
0=E_{3}^{\prime} \cdot \zeta_{*}\left(\widetilde{C}_{1}\right)=E_{3} \cdot \widetilde{C}_{1}+m_{1} E_{1} \cdot \widetilde{C}_{1}+m_{2} E_{2} \cdot \widetilde{C}_{1}=1-m_{1}
$$

so $m_{1}=1$, and similarly $m_{2}=1$. Hence

$$
E_{3}^{\prime} \cdot C_{3}^{\prime}=\left(E_{3}+E_{1}+E_{2}\right) \cdot \widetilde{C}_{3}=1 .
$$

This says that $C_{3}^{\prime}$ is a minimal element in the extremal ray $\beta$, namely that, for every curve $C \subset X_{0}$ such that $[C] \in \beta$, we have $C \equiv m C_{3}^{\prime}$ with $m \in \mathbb{Z}_{>0}$.

Moreover

$$
K_{X}=\varphi_{1}^{*}\left(K_{W_{1}}\right)+E_{1}=\zeta^{*}\left(K_{X_{0}}\right)+E_{1}+E_{2},
$$

which gives

$$
-K_{X_{0}} \cdot C_{3}^{\prime}=\zeta^{*}\left(-K_{X_{0}}\right) \cdot \widetilde{C}_{3}=\left(-K_{X}+E_{1}+E_{2}\right) \cdot \widetilde{C}_{3}=3 .
$$

This says that the length $l(\beta)$ of the extremal ray $\beta$, that is the minimal anticanonical degree of rational curves whose class is in $\beta$, is 3. Now [Wiś91a, Theorem 1.1] yields that for every non-trivial 


\section{Casagrande}

fiber $F$ of $h$ we have

$$
\operatorname{dim} F+\operatorname{dim} \operatorname{Locus}(\beta) \geqslant n+2 .
$$

On the other hand $f_{0}$ is finite on fibers of $h$, so $\operatorname{dim} F \leqslant 2$. Therefore $\operatorname{dim} \operatorname{Locus}(\beta)=n$, every fiber of $h$ has dimension 2 , and $\operatorname{dim} W_{0}=n-2$.

Now $f_{0}$ and $h$ induce a finite morphism $X_{0} \rightarrow \mathbb{P}^{2} \times W_{0}$; let $d$ be its degree.

Let $w_{0} \in W_{0}$ be a general point and $S_{0}=h^{-1}\left(w_{0}\right)$. Then $S_{0}$ is a smooth surface and $g:=$ $\left(f_{0}\right)_{\mid S_{0}}: S_{0} \rightarrow \mathbb{P}^{2}$ is finite of degree $d$.

Since in $X$ the divisor $E_{3}$ intersects transversally $E_{1}$ and $E_{2}, \zeta_{\mid E_{3}}$ is an isomorphism, so that $E_{3}^{\prime} \cong \mathbb{P}^{1} \times F$ and $\left(f_{0}\right)_{\mid E_{3}^{\prime}}$ is the first projection.

Observe that $f_{0}\left(E_{3}^{\prime}\right)$ is a line $l$ (the line through $p_{1}$ and $p_{2}$ ), and $h_{\mid E_{3}^{\prime}}: E_{3}^{\prime} \rightarrow W_{0}$ is surjective, so it factors as

$$
E_{3}^{\prime} \stackrel{\pi_{2}}{\longrightarrow} F \stackrel{\xi}{\rightarrow} W_{0}
$$

where $\pi_{2}$ is the second projection and $\xi$ is a finite morphism. We have

$$
g^{-1}(l)=\left(f_{0}\right)^{-1}(l) \cap S_{0}=E_{3}^{\prime} \cap S_{0}=E_{3}^{\prime} \cap h^{-1}\left(w_{0}\right)=\left(h_{\mid E_{3}^{\prime}}\right)^{-1}\left(w_{0}\right) \cong \mathbb{P}^{1} \times \xi^{-1}\left(w_{0}\right) .
$$

On the other hand $g^{-1}(l)$ is the support of an ample divisor in $S_{0}$ and hence it is connected. This implies that $\xi$ is an isomorphism and that $\widetilde{l}:=g^{-1}(l) \simeq \mathbb{P}^{1} \times w_{0}$.

Then $g^{*}(l)=d \widetilde{l}$; on the other hand $g_{\widetilde{l}}=\left(f_{0}\right)_{\widetilde{l}}$ is an isomorphism, so $g_{*}(\widetilde{l})=l$. Now we have

$$
1=l^{2}=l \cdot g_{*}(\widetilde{l})=g^{*}(l) \cdot \widetilde{l}=d(\widetilde{l})^{2},
$$

so $d=1$ and we are done; Theorem 1.1(i) is proved.

\section{Fano manifolds with a quasi-elementary contraction onto a 3-fold}

In this section we prove Theorem 1.1(ii). Let us consider a smooth Fano variety $X$ of dimension $n \geqslant 4$, and a quasi-elementary contraction $f: X \rightarrow Y$ with $\operatorname{dim} Y=3$.

Recall that $Y$ is factorial and has at most isolated canonical singularities by Lemma 3.10(i) and (iv). Let $\psi: Y \rightarrow Z$ be an elementary contraction of $Y$. We consider all possibilities for $\psi$.

If $\psi$ is of type $(3,0)$, then $Z$ is a point and $Y$ is Fano with $\rho_{Y}=1$.

If $\psi$ is of type $(3,1)$, then $Z \simeq \mathbb{P}^{1}$ and $\rho_{Y}=2$. In this case the other elementary contraction of $Y$ cannot be again of type $(3,1)$, because non-trivial fibers of distinct elementary contractions can intersect at most in finitely many points, and $Y$ is factorial.

Claim 6.1. If $\psi: Y \rightarrow Z$ is of type $(3,2)$, then $\psi$ is equidimensional, and we have the following possibilities:

(i) $\rho_{Y}=2$ and $Z \cong \mathbb{P}^{2}$;

(ii) $\rho_{Y}=3$ and $Z$ is either $\mathbb{P}^{1} \times \mathbb{P}^{1}$ or $\mathbb{F}_{1}$; or

(iii) $Z$ is a smooth Del Pezzo surface with $\rho_{Z} \geqslant 3, Y \cong Z \times \mathbb{P}^{1}, \psi$ is the projection, and $X \cong Z \times W$, where $W$ is a smooth Fano variety of dimension $n-2$ with a quasi-elementary contraction onto $\mathbb{P}^{1}$.

Proof. We have $\operatorname{dim} Z=2$ and $\psi \circ f: X \rightarrow Z$ is quasi-elementary (see Example 3.2). By Theorem 1.1(i), $\psi \circ f$ is equidimensional and $Z$ is a smooth Del Pezzo surface. Then $\psi$ must be equidimensional too.

If $\rho_{Z}=1,2$, we get the first two cases. If $\rho_{Z} \geqslant 3$, again by Theorem 1.1 (i) we see that $X \cong Z \times W$, where $W$ is a smooth Fano variety of dimension $n-2$, and $\psi \circ f$ is the projection onto $Z$. Then 


\section{QUASI-ELEMENTARY CONTRACTIONS OF FANO MANIFOLDS}

any intermediate contraction $Z \times W \rightarrow V \rightarrow Z$ must be onto a product $V \cong Z \times F$, hence we get $Y \cong Z \times \mathbb{P}^{1}$. Moreover, $f$ induces a contraction $g: W \rightarrow \mathbb{P}^{1}$. Since $f$ is quasi-elementary, it is easy to see that $g$ is quasi-elementary too.

Claim 6.2. If $\psi$ is of type $(2,0)$, then we have the following possibilities:

(i) $\rho_{Y}=2$ and $Y$ has an elementary contraction of type $(3,2)$; or

(ii) $\rho_{Y}=3$ and $Y$ has a birational elementary contraction with fibers of dimension at most 1 .

Proof. The divisor $\operatorname{Exc}(\psi)$ satisfies $\operatorname{dim} \mathcal{N}_{1}(\operatorname{Exc}(\psi), Y)=1$, hence Proposition 3.16 yields $\rho_{Y} \leqslant 3$. However, $Y$ has a non-trivial elementary contraction, hence $\rho_{Y}>1$. Then the statement follows from Remark 3.17.

Claim 6.3. Let $\psi: Y \rightarrow Z$ be an elementary contraction that is birational with fibers of dimension at most 1 . Then $\psi$ is the blow-up of a smooth curve $C \subset Z_{\text {reg }}$, and we have a commutative diagram

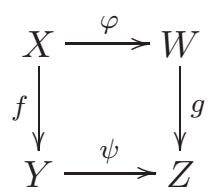

where $W$ is smooth, $\varphi$ is the blow-up of a smooth subvariety of codimension $2, \operatorname{Exc}(\varphi)=f^{*}(\operatorname{Exc}(\psi))$, and $g$ is a quasi-elementary Mori contraction.

Proof. Recall that $\psi$ has a lifting as explained in $\S 2.5$, so we have a diagram as above. Since $X$ is Fano, $\varphi$ is a Mori contraction. Then Theorem 3.14 yields the statement.

In particular, Claim 6.3 implies that $Y$ has no small elementary contractions.

Claim 6.4. In the setting of Claim 6.3, suppose that $Y$ is Fano. Set $E:=\operatorname{Exc}(\varphi)$ and $S:=\operatorname{Exc}(\psi)$. Then the following statements are equivalent:

(i) $W$ is not Fano;

(ii) $Z$ is not Fano; and

(iii) $S \cong \mathbb{P}^{1} \times \mathbb{P}^{1}$ with normal bundle $\mathcal{O}_{\mathbb{P}^{1} \times \mathbb{P}^{1}}(-1,-1)$, and there is an extremal ray $\beta$ of $\operatorname{NE}(Y)$, different from $\mathrm{NE}(\psi)$, such that $S \cdot \beta<0$.

Proof. (i) $\Rightarrow$ (ii) If $W$ is not Fano, by [Wiś91a, Proposition 3.4] there exists an extremal ray $\alpha$ of $\mathrm{NE}(X)$ such that $\alpha \neq \mathrm{NE}(\varphi)$ and $\alpha \cdot E<0$. Observe that $\alpha$ is not contracted by $f$, because $E \cdot C=0$ for every curve $C \subset X$ contracted by $f$.

Let $\varphi_{2}: X \rightarrow W_{2}$ be the contraction of $\alpha$ and let $F_{0}$ be an irreducible component of a non-trivial fiber of $\varphi_{2}$. Then $F_{0} \subset E$ and $f$ is finite on $F_{0}$, hence $f\left(F_{0}\right) \subseteq S$ and $\operatorname{dim} F_{0} \leqslant 2$. Moreover, if $\operatorname{dim} F_{0}=2$, then $f\left(F_{0}\right)=S$. This would imply that $\operatorname{dim} \mathcal{N}_{1}(S, Y)=1$, while $\operatorname{dim} \mathcal{N}_{1}(S, Y)=2$. Thus $\operatorname{dim} F_{0}=1$.

Now [Wiś91a, Theorem 1.2] yields that $\varphi_{2}$ is a smooth blow-up with exceptional divisor $E$. Observe that $S$ is a smooth $\mathbb{P}^{1}$-bundle over $\psi(S)$. It is not difficult to see that the $\mathbb{P}^{1}$-bundle $\left(\varphi_{2}\right)_{\mid E}$ induces a second rational fibration on $S$, so that $S \cong \mathbb{P}^{1} \times \mathbb{P}^{1}$. Moreover, if $C \subset E$ is a non-trivial fiber of $\varphi_{2}$, then

$$
-1=C \cdot E=C \cdot f^{*}(S)=f_{*}(C) \cdot S,
$$

and this gives $\mathcal{N}_{S / Y} \cong \mathcal{O}_{\mathbb{P}^{1} \times \mathbb{P}^{1}}(-1,-1)$. In particular, $\psi(S)$ is a curve of anticanonical degree 0 in $Z$, so $Z$ is not Fano.

(ii) $\Rightarrow$ (iii) Suppose that $Z$ is not Fano. Observe that $Y$ and $Z$ may be singular; however they are factorial and $K_{Y}=\psi^{*}\left(K_{Z}\right)+S$. Then reasoning as in [Wiś91a, Proposition 3.4] one gets statement (iii). 


\section{Casagrande}

(iii) $\Rightarrow$ (i) The contraction of $\beta$ is birational with fibers of dimension at most 1 ; let $\varphi_{2}: X \rightarrow W_{2}$ be the smooth blow-up given by Claim 6.3. Then $E=\operatorname{Exc}\left(\varphi_{2}\right)$, and $\varphi_{2} \neq \varphi$. If $C \subset E$ is a non-trivial fiber of $\varphi_{2}$, then $K_{X} \cdot C=E \cdot C=-1$, which yields $-K_{W} \cdot \varphi_{*}(C)=0$. Hence $W$ is not Fano.

Claim 6.5. Suppose that $Y$ is Fano, and let $\beta_{1}$ and $\beta_{2}$ be two extremal rays of $\operatorname{NE}(Y)$ with distinct divisorial loci $S_{i}=\operatorname{Locus}\left(\beta_{i}\right)$. If $S_{1} \cap S_{2} \neq \emptyset$, then the contraction $\psi: Y \rightarrow Z$ of one of the $\beta_{i}$ is the blow-up of a smooth curve $C \subset Z_{\text {reg }}$, and $Z$ is Fano.

Proof. Let $\psi_{i}: Y \rightarrow Z_{i}$ be the contraction of $\beta_{i}$. Since $S_{1} \cap S_{2} \neq \emptyset$ and $Y$ is factorial, we have $\operatorname{dim} S_{1} \cap S_{2}=1$. Thus $\psi_{1}$ and $\psi_{2}$ cannot both be of type $(2,0)$.

So let us assume that $\psi_{1}$ is of type $(2,1)$. If $Z_{1}$ is Fano, we are done. If $Z_{1}$ is not Fano, Claim 6.4 yields the existence of a second extremal ray $\widetilde{\beta}_{1}$ of $\mathrm{NE}(Y)$, distinct from $\beta_{1}$, with $\widetilde{\beta}_{1} \cdot S_{1}<0$. Then $\mathcal{N}_{1}\left(S_{1}, Y\right)$ is generated by $\beta_{1}$ and $\widetilde{\beta}_{1}$, and no other extremal ray of $\mathrm{NE}(Y)$ can be contained in $\mathcal{N}_{1}\left(S_{1}, Y\right)$.

Thus $\psi_{2}$ cannot be of type $(2,0)$. Let us show that $Z_{2}$ must be Fano. If not, by Claim 6.4 there is another extremal ray $\widetilde{\beta}_{2}$ of $\mathrm{NE}(Y)$, distinct from $\beta_{2}$, with $\widetilde{\beta}_{2} \cdot S_{2}<0$. Since $\beta_{2}$ and $\widetilde{\beta}_{2}$ are not contained in $\mathcal{N}_{1}\left(S_{1}, Y\right)$, we have $\beta_{2} \cdot S_{1} \geqslant 0$ and $\widetilde{\beta}_{2} \cdot S_{1} \geqslant 0$.

Now if $C \subset S_{1} \cap S_{2}$ is an irreducible curve, we get $C \cdot S_{1}<0$ because $\beta_{1} \cdot S_{1}<0$ and $\widetilde{\beta}_{1} \cdot S_{1}<0$. On the other hand, $C \cdot S_{1} \geqslant 0$ because $\beta_{2} \cdot S_{1} \geqslant 0$ and $\widetilde{\beta}_{2} \cdot S_{1} \geqslant 0$. Thus we have a contradiction.

Claim 6.6. In the setting of Claim 6.3, suppose that $Y$ is Fano. Then we are in one of the following situations:

(i) $W$ and $Z$ are Fano;

(ii) $\rho_{Y} \geqslant 3$ and $Y$ has another elementary contraction $\widetilde{\psi}$ as in Claim 6.3, such that the corresponding $\widetilde{W}, \widetilde{Z}$ are Fano; or

(iii) $\rho_{Y}=3$ and $Y$ has an elementary contraction of type $(3,2)$.

Proof. Let us assume that we are not in situation (i), so $W$ and $Z$ are not Fano. By Claim 6.4 we have $S \cong \mathbb{P}^{1} \times \mathbb{P}^{1}$, and there is a second extremal ray $\beta$ of $\mathrm{NE}(Y)$ with $\beta \cdot S<0$.

There exists an extremal ray $\widetilde{\beta}$ of $\mathrm{NE}(Y)$ such that $S \cdot \widetilde{\beta}>0$; let $\widetilde{\psi}: Y \rightarrow \widetilde{Z}$ be its contraction.

Clearly $\widetilde{\beta}$ is distinct from $\mathrm{NE}(\psi)$ and $\beta$, and the elements of three distinct extremal rays must by linearly independent in $\mathcal{N}_{1}(Y)$. Hence $\rho_{Y} \geqslant 3$ and $\widetilde{\beta} \cap \mathcal{N}_{1}(S, Y)=\{0\}$, which implies that $\widetilde{\psi}$ is finite on $S$. On the other hand, $S$ must intersect every non-trivial fiber of $\widetilde{\psi}$, hence $\widetilde{\psi}$ has fibers of dimension at most 1 .

If $\widetilde{\psi}$ is of fiber type, then it is of type $(3,2)$. Let us consider

$$
\left(\widetilde{\psi}_{*}\right)_{\mid \mathcal{N}_{1}(S, Y)}: \mathcal{N}_{1}(S, Y) \longrightarrow \mathcal{N}_{1}(\widetilde{Z}) .
$$

Since ker $\widetilde{\psi}_{*}$ is the line spanned by $\widetilde{\beta}$ in $\mathcal{N}_{1}(Y)$, we have $\operatorname{ker} \widetilde{\psi}_{*} \cap \mathcal{N}_{1}(S, Y)=\{0\}$ and $\left(\widetilde{\psi}_{*}\right)_{\mid \mathcal{N}_{1}(S, Y)}$ is injective. On the other hand, $\widetilde{\psi}(S)=\widetilde{Z}$, hence $\left(\widetilde{\psi}_{*}\right)_{\mid \mathcal{N}_{1}(S, Y)}$ is surjective too. This gives $\rho_{\widetilde{Z}}=2$ and $\rho_{Y}=3$, and thus situation (iii).

Suppose that $\widetilde{\psi}$ is birational. Then $\operatorname{Exc}(\widetilde{\psi}) \cap \operatorname{Exc}(\psi) \neq \emptyset$, and Claim 6.5 implies that $\widetilde{Z}$ is Fano. Finally also $\widetilde{W}$ is Fano by Claim 6.4 , and we get situation (ii).

Claim 6.7. Let $\pi: Y \rightarrow T$ be a contraction onto a surface. Let $\alpha_{1}, \ldots, \alpha_{m}$ be the extremal rays of $\operatorname{NE}(\pi)$ and $\psi_{i}: Y \rightarrow Z_{i}$ be the contraction of $\alpha_{i}$. Then $\operatorname{Exc}\left(\psi_{i}\right) \cap \operatorname{Exc}\left(\psi_{j}\right) \neq \emptyset$ for some $i \neq j$.

Proof. Set $S_{i}:=\operatorname{Exc}\left(\psi_{i}\right)$, and assume by contradiction that $S_{i} \cap S_{j}=\emptyset$ for every $i \neq j$. Then each $\psi_{i}$ is birational and $S_{i} \cdot \alpha_{i}<0$ for every $i$ by Remark 2.4; moreover $S_{i} \cdot \alpha_{j}=0$ for every $i \neq j$. 


\section{QUASI-ELEMENTARY CONTRACTIONS OF FANO MANIFOLDS}

Let $C \subset Y$ be an irreducible curve that is contracted by $\pi$ but not contained in $S_{1} \cup \cdots \cup S_{m}$. Then

$$
C \equiv \sum_{i=1}^{m} \lambda_{i} l_{i} \quad \text { where }\left[l_{i}\right] \in \alpha_{i} \text { and } \lambda_{i} \in \mathbb{Q} \geqslant 0 \text { for every } i=1, \ldots, m .
$$

So $0 \leqslant C \cdot S_{i}=\lambda_{i}\left(l_{i} \cdot S_{i}\right)$ implies that $\lambda_{i}=0$ for every $i=1, \ldots, m$, a contradiction.

Proof of Theorem 1.1(ii). Let us suppose that $\rho_{Y} \geqslant 4$. By Claims 6.1, 6.2, and 6.3, any elementary contraction of $Y$ is either a smooth blow-up or a $\mathbb{P}^{1}$-bundle. This implies that $Y$ is Fano (but possibly singular) by Remark 3.11 .

Suppose now that $Y$ is smooth and that $\rho_{Y} \geqslant 6$. Then $Y \cong S \times \mathbb{P}^{1}$ with $S$ a Del Pezzo surface by [MM81, Theorem 2]. Thus $X \rightarrow S$ is a quasi-elementary contraction (see Example 3.2), and applying Theorem 1.1(i) as in the proof of Claim 6.1 we easily get the statement.

Thus we are left to prove that if $\rho_{Y} \geqslant 4$, then $Y$ must be smooth.

By contradiction, assume that $Y$ is singular and $\rho_{Y} \geqslant 4$. By Claims 6.1, 6.2, and 6.6, we can construct a sequence

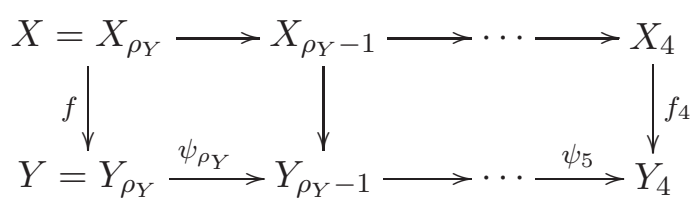

where, for every $i=4, \ldots, \rho_{Y}, X_{i}$ is smooth and Fano, $f_{i}$ is a quasi-elementary contraction, $Y_{i}$ is Fano with $\rho_{Y_{i}}=i$, and $\psi_{i}$ is the blow-up of a smooth curve contained in the smooth locus of $Y_{i-1}$. In particular, each $Y_{i}$ is singular, because $Y$ is.

Since $Y_{4}$ is singular and $\rho_{Y_{4}}=4$, Claim 6.1 implies that $Y_{4}$ has no elementary contraction of fiber type.

Claim 6.8. The 3-fold $Y_{4}$ has no contraction onto a surface.

Proof. By contradiction let $\pi: Y_{4} \rightarrow T$ be such a contraction. Then $\pi$ cannot be elementary, so $\rho_{T} \leqslant 2$.

The cone $\mathrm{NE}(\pi)$ contains $m \geqslant 2$ extremal rays, whose contractions are birational. Call $S_{1}, \ldots, S_{m}$ their exceptional loci. By Claim 6.7 they cannot all be disjoint, so we can assume that $S_{1} \cap S_{2} \neq \emptyset$.

Using Claim 6.5, we can assume that the elementary contraction $\psi_{4}: Y_{4} \rightarrow Y_{3}$ with exceptional locus $S_{1}$ is the blow-up of a smooth curve $C_{3} \subset\left(Y_{3}\right)_{\text {reg }}$, and that $Y_{3}$ is Fano. We observe that $Y_{3}$ is singular because $Y_{4}$ is. As before we get

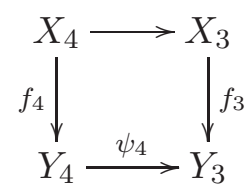

where $X_{3}$ is smooth and Fano and $f_{3}$ is a quasi-elementary contraction. Moreover, $\pi$ induces a contraction $\pi^{\prime}: Y_{3} \rightarrow T$.

If $\pi^{\prime}$ is elementary, then $\rho_{T}=2, \pi^{\prime}$ is equidimensional, and $T$ is either $\mathbb{P}^{1} \times \mathbb{P}^{1}$ or $\mathbb{F}_{1}$ by Claim 6.1. Hence $\pi^{\prime}$ is a conic bundle (see [Sar82, $\S 1$ ] for the definition and properties of conic bundles). Let $\Delta_{\pi^{\prime}} \subset T$ be the discriminant locus of $\pi^{\prime}$, which is non-empty because $Y_{3}$ is singular.

We recall that $C_{3} \subset Y_{3}$ cannot intersect any curve of anticanonical degree 1 by Remark 2.3. Thus $C_{3}$ cannot be a component of a reducible fiber of $\pi^{\prime}$.

If $C_{3}$ is an irreducible fiber, let $p=\pi^{\prime}\left(C_{3}\right) \in T$ and call $T^{\prime}$ the blow-up of $T$ in $p$ : then $Y_{4}$ has an elementary contraction onto $T^{\prime}$, which is impossible. 


\section{Casagrande}

Hence $\pi^{\prime}\left(C_{3}\right)$ is a curve in $T$, and it is disjoint from $\Delta_{\pi^{\prime}}$ because $C_{3}$ cannot intersect singular fibers of $\pi^{\prime}$.

If $T \cong \mathbb{P}^{1} \times \mathbb{P}^{1}$, the only possibility is that $\Delta_{\pi^{\prime}}$ is a union of fibers of a projection $\mathbb{P}^{1} \times \mathbb{P}^{1} \rightarrow \mathbb{P}^{1}$, hence a disjoint union of smooth rational curves. But this is impossible, see for instance [Pro05, Lemma 5.3].

Consider now the case where $T \cong \mathbb{F}_{1}$. Then we have a contraction $Y_{3} \rightarrow \mathbb{F}_{1} \rightarrow \mathbb{P}^{2}$, which has a second factorization $Y_{3} \rightarrow Y_{2} \rightarrow \mathbb{P}^{2}$. It is not difficult to see that $\psi_{3}: Y_{3} \rightarrow Y_{2}$ is birational with fibers of dimension at most 1 , so by Claim 6.3 it is again a blow-up of a smooth curve $C_{2} \subset\left(Y_{2}\right)_{\text {reg }}$, and we have the diagram

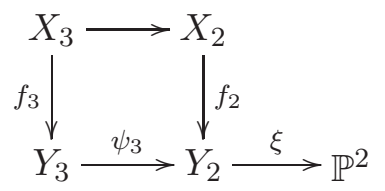

where $X_{2}$ is smooth, $Y_{2}$ is singular with $\rho_{Y_{2}}=2$, and $f_{2}$ is a quasi-elementary Mori contraction.

The only curve in $Y_{2}$ which could have non-positive anticanonical degree is $C_{2}$. Thus $\xi: Y_{2} \rightarrow \mathbb{P}^{2}$ is a conic bundle with non-empty discriminant locus $\Delta_{\xi} \subset \mathbb{P}^{2}$. If $\xi\left(C_{2}\right)$ is a curve in $\mathbb{P}^{2}$, then $C_{2}$ must intersect some singular fiber, which is again impossible by Remark 2.3. Thus $C_{2}$ is a smooth fiber of $\xi, Y_{2}$ is Fano, and $X_{2}$ is Fano too by Claim 6.4.

Let us consider the other elementary contraction $\eta: Y_{2} \rightarrow Z$ of $Y_{2}$.

If $\eta$ is again of type $(3,2)$, reasoning as above we get that $\eta$ must contract $C_{2}$, a contradiction because $\mathrm{NE}(\eta) \cap \mathrm{NE}(\xi)=\{0\}$.

If $\eta$ is of type $(3,1)$, it is a fibration in Del Pezzo surfaces over $\mathbb{P}^{1}$. Then $Y_{2}$ is a finite cover of $\mathbb{P}^{1} \times \mathbb{P}^{2}$, and $C_{2}$ is the inverse image of $\mathbb{P}^{1} \times\{p t\}$. This implies that $Y_{3}$ is a finite cover of $\mathbb{P}^{1} \times \mathbb{F}_{1}$, which gives a surjective morphism $Y_{3} \rightarrow \mathbb{P}^{1} \times \mathbb{P}^{1}$. Taking the Stein factorization we get an elementary contraction $Y_{3} \rightarrow T^{\prime}$ of type $(3,2)$, where $T^{\prime}$ is a finite cover of $\mathbb{P}^{1} \times \mathbb{P}^{1}$. By Claim $6.1, T^{\prime}$ can be $\mathbb{P}^{1} \times \mathbb{P}^{1}$ or $\mathbb{F}_{1}$, but $T^{\prime}$ has two distinct fibrations, thus $T^{\prime} \cong \mathbb{P}^{1} \times \mathbb{P}^{1}$. We have already excluded this possibility.

Therefore, $\eta$ is birational; let $E$ be its exceptional divisor. Since $E \cdot \operatorname{NE}(\eta)<0$ by Remark 2.4, it must be $E \cdot \mathrm{NE}(\xi)>0$, hence $\xi(E)=\mathbb{P}^{2}$ and $E$ intersects $C_{2}$. In particular, $E$ cannot be covered by curves of anticanonical degree 1 , thus $\eta$ is of type $(2,0)$. Moreover, $E \cdot C_{2} \geqslant 2$, because $\xi$ has singular fibers.

The composite contraction $Y_{3} \rightarrow Z$ has a second factorization

$$
Y_{3} \stackrel{\sigma}{\rightarrow} \tilde{Y}_{2} \stackrel{\chi}{\rightarrow} Z
$$

It is not difficult to see that $\sigma$ has exceptional locus $\psi_{3}^{*}(E)$ and is the blow-up of a smooth curve

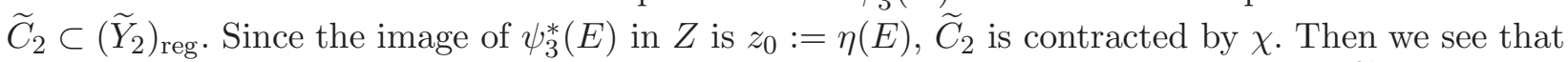
$\chi$ is again a Mori contraction of type $(2,1)$ with exceptional divisor $\sigma\left(\operatorname{Exc}\left(\psi_{3}\right)\right)$, and $\widetilde{Y}_{2}$ is Fano. In particular, $\chi$ is again a blow-up of a smooth curve contained in $Z_{\text {reg }}$, so that $z_{0}$ is a smooth point. Moreover, $\psi_{3}^{*}(E)$ is contained in the smooth locus of $Y_{3}$, so that $E \subset\left(Y_{2}\right)_{\mathrm{reg}}$.

Therefore $\eta$ is just the blow-up of $z_{0}$ and $E \cong \mathbb{P}^{2}$. If $E$ intersects $C_{2}$ in at least two distinct points, take $l$ to be a line in $E$ through these two points. Let $\widetilde{l}$ be the proper transform of $l$ in $Y_{3}$. Then $-K_{Y_{2}} \cdot l=2$ and $\widetilde{l} \cdot \operatorname{Exc}\left(\psi_{3}\right) \geqslant 2$, so by Remark 2.3 we get $-K_{Y_{3}} \cdot \widetilde{l} \leqslant 0$, a contradiction.

If $E$ intersects $C_{2}$ in a single non-reduced point $y_{0}$, similarly as before take $l$ to be a line in $E \cong \mathbb{P}^{2}$ through $y_{0}$. Then the schematic intersection $\psi_{3}^{*}(E) \cap \operatorname{Exc}\left(\psi_{3}\right)$ is non-reduced along the fiber of $\psi_{3}$ over $y_{0}$, thus again $\widetilde{l} \cdot \operatorname{Exc}\left(\psi_{3}\right) \geqslant 2$ gives a contradiction. This concludes the case where $\rho_{T}=2$.

We still have to exclude the case where $\rho_{T}=1$ and $Y_{3}$ has no elementary contractions of type $(3,2)$. Reasoning as for $Y_{4}$, we see that one of the two extremal rays of $\mathrm{NE}\left(\pi^{\prime}\right)$ yields a blow-up 


\section{QUASI-ELEMENTARY CONTRACTIONS OF FANO MANIFOLDS}

$\psi_{3}: Y_{3} \rightarrow Z_{2}$ of a smooth curve $C^{\prime} \subset\left(Z_{2}\right)_{\text {reg }}$, and $Z_{2}$ is Fano with $\rho_{Z_{2}}=2$. Now $\pi^{\prime}$ yields an elementary contraction $\pi^{\prime \prime}: Z_{2} \rightarrow T$. Claim 6.1 gives $T \cong \mathbb{P}^{2}$, and $\Delta_{\pi^{\prime \prime}}$ is non-empty. As before we easily get a contradiction. This completes the proof of Claim 6.8.

Using Claims 6.8, 6.2, and 6.6, we get a sequence

$$
Y_{4} \stackrel{\psi_{4}}{\longrightarrow} Y_{3} \stackrel{\psi_{3}}{\longrightarrow} Y_{2} \stackrel{\psi_{2}}{\longrightarrow} Y_{1}
$$

where each $Y_{i}$ is Fano and each $\psi_{i}$ is the blow-up of a smooth curve $C_{i-1} \subset\left(Y_{i-1}\right)_{\text {reg. }}$. In particular $Y_{1}$ is factorial with isolated canonical singularities, singular, and $\rho_{Y_{1}}=1$.

We first show that $Y_{1}$ must have terminal singularities, using the following lemma.

Lemma 6.9. Let $Z$ be a three-dimensional $\mathbb{Q}$-factorial projective variety, with isolated canonical singularities and $K_{Z}$ Cartier. Suppose that $Z$ is Fano with $\rho_{Z}=1$, and that the singularities of $Z$ are not terminal. Then one of the following occurs:

(i) $Z$ contains a one-dimensional family of curves of anticanonical degree 1 passing through a singular point; or

(ii) $Z$ is covered by a family of curves of anticanonical degree at most 2 passing through a singular point.

We postpone the proof of Lemma 6.9 and carry on with the proof of Theorem 1.1(ii).

Suppose that $Y_{1}$ has at least one non-terminal singular point: then Lemma 6.9 applies to $Y_{1}$. If part (i) holds, let $S \subset Y_{1}$ be a surface covered by curves of anticanonical degree 1 . Since $\rho_{Y_{1}}=1$, $S$ is ample, and $C_{1} \cap S \neq \emptyset$. Observe that, even if $C_{1} \subset S, C_{1}$ does not contain any singular point, hence it cannot be a member of the family given by part (i). Thus $C_{1}$ intersects some curve of anticanonical degree 1, which is impossible by Remark 2.3 .

Suppose now that part (ii) holds for $Y_{1}$. If $C_{1}$ is a component of some reducible curve $l_{1}$ of the family, it must be $l_{1}=C_{1} \cup C_{1}^{\prime}$ with $-K_{Y_{1}} \cdot C_{1}=-K_{Y_{1}} \cdot C_{1}^{\prime}=1$, which again gives a contradiction. Again $C_{1}$ cannot be a member of the family, because it does not contain singular points. Hence $C_{1}$ is not contained in any member of the family; let $T$ be an irreducible surface containing $C_{1}$ such that through every point of $C_{1}$ there is a curve of anticanonical degree at most 2 contained in $T$. Let $\widetilde{T}$ be the proper transform of $T$ in $Y_{2}$. Then through every point of $\widetilde{T} \cap \operatorname{Exc}\left(\psi_{2}\right)$ there is a curve of anticanonical degree 1 contained in $\widetilde{T}$ (see Remark 2.3).

Consider $C_{2} \subset\left(Y_{2}\right)_{\mathrm{reg}}$. If $\psi_{2}\left(C_{2}\right)$ is a point, then $C_{2}$ must intersect some curve of anticanonical degree 1 contained in $\widetilde{T}$. On the other hand, if $\psi_{2}\left(C_{2}\right)$ is a curve, then it must intersect $T$, thus $C_{2}$ must intersect $\psi_{2}^{-1}(T)=\widetilde{T} \cup \operatorname{Exc}\left(\psi_{2}\right)$. In any case $C_{2}$ will intersect some curve of anticanonical degree 1 , which gives a contradiction.

Hence $Y_{1}$ has terminal singularities, and the same holds for each $Y_{i}$. Consider in particular $Y_{4}$. By [Nam97], $Y_{4}$ has a smoothing, that is an integral complex space $\mathcal{Y}$, with a projective flat morphism $\mathcal{Y} \rightarrow \Delta$ onto the complex unit disk, such that $Y_{4}$ is the fiber over 0 while the fiber $Y_{t}$ over $t \neq 0$ is a smooth Fano 3-fold. It is proven in [JR06] that $\operatorname{Pic} Y_{4} \cong \operatorname{Pic} Y_{t}$, in particular $\rho_{Y_{t}}=4$. Then we know by Mori and Mukai's classification that $Y_{t}$ has a conic bundle structure (not necessarily elementary), see [IP99, Theorem on p. 141]. Our goal is to deduce from this that $Y_{4}$ must have a contraction onto a surface, contradicting Claim 6.8.

For this we need the following lemma, based on [JR06].

Lemma 6.10. Let $Z$ be a three-dimensional factorial Fano variety with terminal singularities and $\mathcal{Z} \rightarrow \Delta$ a smoothing. Consider the inclusions $i_{t}: Z_{t} \hookrightarrow \mathcal{Z}$ and $i_{0}: Z_{0} \hookrightarrow \mathcal{Z}$. 


\section{Casagrande}

Then the push-forwards $\left(i_{t}\right)_{*}$ and $\left(i_{0}\right)_{*}$ induce bijections among the cones

$$
\operatorname{NE}\left(Z_{t}\right), \quad \mathrm{NE}(\mathcal{Z} / \Delta), \quad \text { and } \operatorname{NE}\left(Z_{0}\right),
$$

and every contraction of $Z_{t}$ or of $Z_{0}$ is the restriction of the contraction of the corresponding face of $\mathrm{NE}(\mathcal{Z} / \Delta)$.

By Lemma 6.10, the conic bundle on $Y_{t}$ induces a contraction $\mathcal{Y} \rightarrow \mathcal{T} \rightarrow \Delta$. This restricts to a contraction $\pi: Y_{4} \rightarrow T$ onto a surface, which contradicts Claim 6.8. Theorem 1.1(ii) is proved, and this concludes the proof of Theorem 1.1.

Proof of Lemma 6.9. Let $\psi: Z^{\prime} \rightarrow Z$ be a partial crepant resolution such that $Z^{\prime}$ has terminal and factorial singularities (see $[\mathrm{KM} 98, \S 6.3]$ ). Hence $K_{Z^{\prime}}=\psi^{*}\left(K_{Z}\right),-K_{Z^{\prime}}$ is nef and big, and $\rho_{Z^{\prime}}>1$ because $\psi$ is not an isomorphism.

Since $Z$ is $\mathbb{Q}$-factorial, for every non-terminal point $p \in Z$ the inverse image $\psi^{-1}(p)$ has pure dimension 2, therefore $\operatorname{Exc}(\psi)$ is a divisor. Moreover, any irreducible curve contained in $\operatorname{Exc}(\psi)$ has anticanonical degree 0 .

There is at least one elementary Mori contraction $f: Z^{\prime} \rightarrow W$. Observe that $f$ must be finite on $\operatorname{Exc}(\psi)$, because any curve contracted by $f$ has positive anticanonical degree. Hence any fiber $F$ of $f$ such that $F \cap \operatorname{Exc}(\psi) \neq \emptyset$ has dimension at most 1 .

Suppose that $f$ is of fiber type. Then it must be of type $(3,2)$ and [Cut88, Theorem 7] says that $W$ is a smooth surface and $f$ is a conic bundle. Moreover, if $E \subseteq \operatorname{Exc}(\psi)$ is an irreducible component, then $f(E)=W$, so that every fiber of $f$ intersects $E$. Then the fibers of $f$ give a covering family of curves of anticanonical degree at most 2 in $Z$, all passing through the singular point $\psi(E)$, and we get part (ii).

Suppose that $f$ is birational. Since $Z^{\prime}$ is Gorenstein, $f$ is divisorial; set $D:=\operatorname{Exc}(f)$. We claim that $D$ cannot be disjoint from $\operatorname{Exc}(\psi)$. In fact, if so, $\psi(D)$ would be a non-nef Cartier divisor in $Z$, which is impossible because $\rho_{Z}=1$.

Hence $f$ must be of type $(2,1)$ and by [Cut88, Theorem 4] we have $-K_{Z^{\prime}} \cdot l=1$ for the general fiber $l$ of $f$. Again, if $E$ is an irreducible component of $\operatorname{Exc}(\psi)$ intersecting $D$, every fiber $l$ of $f$ must intersect $E$. So we get a one-dimensional family of curves of anticanonical degree 1 in $Z$, passing through the singular point $\psi(E)$.

Proof of Lemma 6.10. By [JR06, Proposition 1.1], $\mathcal{Z}$ has at most isolated terminal factorial singularities at the singular points of $Z_{0}$.

We refer to [KMM87, $\S 0-1]$ for the notation in the relative situation. Observe that for a projective morphism in the analytic category the standard results of the Minimal Model Program hold, see [KM98, Example 2.17] and references therein. In particular, since each fiber of $\mathcal{Z} \rightarrow \Delta$ is Fano, $\mathrm{NE}(\mathcal{Z} / \Delta)$ is closed and polyhedral by the relative version of the cone theorem.

We first observe that the linear maps

$$
\left(i_{t}\right)_{*}: \mathcal{N}_{1}\left(Z_{t}\right) \longrightarrow \mathcal{N}_{1}(\mathcal{Z} / \Delta) \text { and }\left(i_{0}\right)_{*}: \mathcal{N}_{1}\left(Z_{0}\right) \longrightarrow \mathcal{N}_{1}(\mathcal{Z} / \Delta)
$$

are isomorphisms. In fact they are dual to the restrictions

$$
\operatorname{Pic}(\mathcal{Z}) \otimes \mathbb{R} \longrightarrow \operatorname{Pic}\left(Z_{t}\right) \otimes \mathbb{R} \quad \text { and } \operatorname{Pic}(\mathcal{Z}) \otimes \mathbb{R} \longrightarrow \operatorname{Pic}\left(Z_{0}\right) \otimes \mathbb{R}
$$

which are isomorphism by [JR06, Theorem 1.4]. Moreover, we have $\left(i_{t}\right)_{*} \mathrm{NE}\left(Z_{t}\right) \subseteq \mathrm{NE}(\mathcal{Z} / \Delta)$ and $\left(i_{0}\right)_{*} \mathrm{NE}\left(Z_{0}\right) \subseteq \mathrm{NE}(\mathcal{Z} / \Delta)$.

Up to shrinking $\Delta$, we can assume that for every extremal ray $\alpha$ of $\mathrm{NE}(\mathcal{Z} / \Delta)$, either $\operatorname{Locus}(\alpha)$ dominates $\Delta$, or $\operatorname{Locus}(\alpha)$ is contained in $Z_{0}$. Let us show that the second case cannot happen. 


\section{QUASI-ELEMENTARY CONTRACTIONS OF FANO MANIFOLDS}

Assume by contradiction that $\operatorname{Locus}(\alpha)$ is contained in $Z_{0}$ and consider the contraction $\varphi$ of $\alpha$, as below.

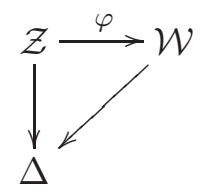

Then $\varphi$ is an isomorphism outside the central fiber, and restricts to a contraction $\varphi_{0}: Z_{0} \rightarrow W_{0}$ on the central fiber. The morphism $\mathcal{W} \rightarrow \Delta$ is projective and the general fiber has dimension 3 , hence $\operatorname{dim} W_{0}=3$ and $\varphi_{0}$ is birational.

This means that $E=\operatorname{Exc}(\varphi)=\operatorname{Exc}\left(\varphi_{0}\right)$ has dimension at most 2, namely $\varphi$ is a small contraction of $\mathcal{Z}$ and it must be of type $(2,0)$. Hence $\mathcal{W}$ is not factorial at $\varphi(E)$.

Since $\left(i_{0}\right)_{*}$ is injective, $\varphi_{0}$ is an elementary contraction of type $(2,0)$ of $Z_{0}$, and $E$ is irreducible. Moreover, $W_{0}$ has terminal singularities and is Fano.

If $W_{0}$ were Gorenstein, $\mathcal{W}$ should be factorial again by [JR06, Proposition 1.1], which is not the case: so $K_{W_{0}}$ is not Cartier. The possibilities for $\varphi_{0}$ are given in [Cut88, Theorem 5], and the only case where $K_{W_{0}}$ is not Cartier is $E \cong \mathbb{P}^{2}$ with normal bundle $\mathcal{N}_{E / Z_{0}} \cong \mathcal{O}_{\mathbb{P}^{2}}(-2)$.

Since $E$ is a smooth prime divisor in the factorial variety $Z_{0}$, it is contained in $\left(Z_{0}\right)_{\text {reg }}$ and hence in $\mathcal{Z}_{\text {reg. Now }}$ Kaw89, Theorem 2.1] yields $\mathcal{N}_{E / \mathcal{Z}} \cong \mathcal{O}_{\mathbb{P}^{2}}(-1)^{\oplus 2}$, a contradiction.

Hence $\operatorname{Locus}(\alpha)$ dominates $\Delta$, which means that $\alpha$ is contained in both cones $\left(i_{t}\right)_{*} \mathrm{NE}\left(Z_{t}\right)$ and $\left(i_{0}\right)_{*} \mathrm{NE}\left(Z_{0}\right)$. Repeating this for every extremal ray of $\mathrm{NE}(\mathcal{Z} / \Delta)$, we get the statement.

\section{Applications and examples}

In this section we prove the corollaries stated in the introduction, and some other applications. We also give some related examples.

Suppose that $X$ is a smooth Fano variety with a quasi-elementary contraction $f: X \rightarrow Y$. If $X$ has other suitable contractions, one can use Theorems 4.1 and 4.4 to get a quasi-elementary contraction $h: X \rightarrow Z$ with $\operatorname{dim} Z \leqslant 3$, and then apply Theorem 1.1. Corollaries 1.4, 7.2, and 7.3 are obtained in this way.

Proof of Corollary 1.4. Recall that $\rho_{X}-\rho_{Y_{i}} \leqslant \rho_{F_{i}}$ because $f_{i}$ is quasi-elementary. Suppose that $\operatorname{dim} F_{1}+\operatorname{dim} F_{2} \geqslant n-2$. By Theorem 4.4(i) and Remark 4.5, there exists a contraction $h: X \rightarrow Z$ where $\operatorname{dim} Z \leqslant n-\left(\operatorname{dim} F_{1}+\operatorname{dim} F_{2}\right)$ and

$$
\rho_{X}-\rho_{Z}=\left(\rho_{X}-\rho_{Y_{1}}\right)+\left(\rho_{X}-\rho_{Y_{2}}\right) \leqslant \rho_{F_{1}}+\rho_{F_{2}} .
$$

This immediately gives $\operatorname{dim} F_{1}+\operatorname{dim} F_{2} \leqslant n$, and, if equality holds, $Z$ is a point so $\rho_{Z}=0$. Moreover, $\operatorname{dim} F_{1}+\operatorname{dim} F_{2}=n-1$ implies that $Z \cong \mathbb{P}^{1}$ and $\rho_{Z}=1$.

Let us notice that $h$ is quasi-elementary too. In fact let $G$ be a general fiber of $h$, then $G$ contains general fibers of $f_{1}$ and $f_{2}$. Since both $f_{i}$ are quasi-elementary, $\mathcal{N}_{1}(G, X)$ contains both $\operatorname{ker}\left(f_{1}\right)_{*}$ and $\operatorname{ker}\left(f_{2}\right)_{*}$. On the other hand we have

$$
\mathcal{N}_{1}(G, X) \subseteq \operatorname{ker} h_{*}=\operatorname{ker}\left(f_{1}\right)_{*}+\operatorname{ker}\left(f_{2}\right)_{*}
$$

as shown in the proof of Theorem 4.4 , so $\mathcal{N}_{1}(G, X)=\operatorname{ker} h_{*}$ and $h$ is quasi-elementary.

Hence if $\operatorname{dim} F_{1}+\operatorname{dim} F_{2}=n-2$ then $Z$ is a Del Pezzo surface by Theorem 1.1(i), so that $\rho_{Z} \leqslant 9$.

Finally, suppose that $\operatorname{dim} F_{1}+\operatorname{dim} F_{2}=n-3$ and that $f_{2}$ is elementary. Similarly to the previous case, Theorem 4.1(i) gives a quasi-elementary contraction $h: X \rightarrow Z$ with $Z \leqslant 3$ and 


\section{Casagrande}

$\rho_{X} \leqslant \rho_{F_{1}}+\rho_{Z}+1$. If $\operatorname{dim} Z \leqslant 2$ we proceed as before. If $\operatorname{dim} Z=3$ then $\rho_{Z} \leqslant 10$ by Theorem 1.1(ii), so we are done.

Remark 7.1. In the statement of Corollary 1.4 one can replace $\rho_{F_{i}}$ by $\operatorname{dim} \operatorname{ker}\left(f_{i}\right)_{*}$, which gives a better bound for instance when $f_{i}$ is elementary. Similarly in the following corollaries.

Corollary 7.2. Let $X$ be a smooth Fano variety and $f: X \rightarrow Y$ a quasi-elementary contraction of fiber type with $\operatorname{dim} Y \geqslant 3$ and general fiber $F$.

Let $\varphi: X \rightarrow W$ be an elementary contraction such that $\mathrm{NE}(\varphi) \cap \mathrm{NE}(f)=\{0\}$. Then every fiber of $\varphi$ has dimension at most $\operatorname{dim} Y$. Moreover:

(i) if $\varphi$ has a fiber of dimension $\operatorname{dim} Y$, then $\rho_{X} \leqslant 1+\rho_{F}$ and $\rho_{Y}=1$; and

(ii) if $\varphi$ has a fiber of dimension $\operatorname{dim} Y-1$, then $\rho_{X} \leqslant 3+\rho_{F}$ and $\rho_{Y} \leqslant 3$.

We state the corollary in this form for completeness; however, let us notice that only the last statement is really new.

Proof. Recall that $\rho_{X} \leqslant \rho_{Y}+\rho_{F}$ because $f$ is quasi-elementary. Since $f$ is finite on fibers of $\varphi$, they have dimension at most $\operatorname{dim} Y$. If there is a fiber with the same dimension as $Y$, let $F_{0}$ be an irreducible component with $\operatorname{dim} F_{0}=\operatorname{dim} Y$. Then $f\left(F_{0}\right)=Y$, so

$$
\left(f_{*}\right)_{\mid \mathcal{N}_{1}\left(F_{0}, X\right)}: \mathcal{N}_{1}\left(F_{0}, X\right) \longrightarrow \mathcal{N}_{1}(Y)
$$

is surjective and $\rho_{Y} \leqslant \operatorname{dim} \mathcal{N}_{1}\left(F_{0}, X\right)=1$.

If $\varphi$ has a fiber of dimension $\operatorname{dim} Y-1$, let $F_{0}$ be an irreducible component with $\operatorname{dim} F_{0}=$ $\operatorname{dim} Y-1$. Then $f\left(F_{0}\right)$ is a prime divisor in $Y$ with $\operatorname{dim} \mathcal{N}_{1}\left(f\left(F_{0}\right), Y\right)=1$, so Proposition 3.16 implies that $\rho_{Y} \leqslant 3$.

Corollary 7.3. Let $X$ be a smooth Fano variety and $f: X \rightarrow Y$ a quasi-elementary contraction of fiber type with $\operatorname{dim} Y \geqslant 3$ and general fiber $F$.

Let $\varphi: X \rightarrow W$ be a divisorial elementary contraction such that $\mathrm{NE}(\varphi) \cap \mathrm{NE}(f)=\{0\}$ and $f(\operatorname{Exc}(\varphi))=Y$.

(i) If the general fiber of $\varphi_{\mid \operatorname{Exc}(\varphi)}$ has dimension $\operatorname{dim} Y-2$, then $\rho_{X} \leqslant \rho_{F}+10$.

(ii) If $f$ is elementary and the general fiber of $\varphi_{\mid \operatorname{Exc}(\varphi)}$ has $\operatorname{dimension} \operatorname{dim} Y-3$, then $\rho_{X} \leqslant 12$.

Proof. The proof is very similar to that of Corollary 1.4. We apply Theorem 4.4(iii) and Theorem 1.1(i) in the first case, Theorem 4.1(iii) and Theorem 1.1(ii) in the second case.

Proof of Corollary 1.2. The first two statements are a direct consequence of Theorem 1.1(i). For the last statement, suppose that $\rho_{X} \geqslant 7$ and that $f: X \rightarrow Y$ is an elementary contraction with $\operatorname{dim} Y=3$. Then $\rho_{Y} \geqslant 6$, so Theorem 1.1(ii) says that $X \cong S \times S^{\prime}$ where $S, S^{\prime}$ are Del Pezzo surfaces and $S^{\prime}$ has an elementary contraction onto $\mathbb{P}^{1}$. Then $S^{\prime} \cong \mathbb{P}^{1} \times \mathbb{P}^{1}$ or $S^{\prime} \cong \mathbb{F}_{1}$.

Proof of Corollary 1.3. The first two statements follow from Corollary 1.4 and Theorem 1.1. Let $f: X \rightarrow Y$ be an elementary contraction with $\operatorname{dim} Y=4$, and $\varphi: X \rightarrow W$ another elementary contraction. If $\varphi$ has a fiber of dimension at least 3 , then $\rho_{X} \leqslant 4$ by Corollary 7.2. In particular, this holds if $\varphi$ is of type $(3,0),(4,0)$, or $(4,1)$. Finally suppose that $f(\operatorname{Exc}(\varphi))=Y$. If $\varphi$ is of fiber type, we have $\rho_{X} \leqslant 12$ by the previous part. If $\varphi$ is birational, then it must be divisorial, so $\rho_{X} \leqslant 12$ by Corollary 7.3 .

Finally we give an application in the spirit of $\S 3.15$. 


\section{QUASI-ELEMENTARY CONTRACTIONS OF FANO MANIFOLDS}

Corollary 7.4. Let $X$ be a smooth Fano variety and $f: X \rightarrow Y$ a non-trivial quasi-elementary contraction of fiber type with general fiber $F$.

Let $D \subset X$ be a prime divisor such that $\operatorname{dim} \mathcal{N}_{1}(D, X)=2$. Then one of the following occurs:

(i) $\rho_{X}=3, Y \simeq \mathbb{P}^{1}$, and $D$ is a fiber of $f$;

(ii) $\rho_{X} \leqslant 4, \rho_{Y} \leqslant 3$, and $f$ is elementary;

(iii) $\rho_{X} \leqslant 10, X \cong F \times Y, Y$ is a Del Pezzo surface, $\rho_{F}=1$, and $\operatorname{dim} f(D)=1$; or

(iv) $\rho_{X} \leqslant 1+\rho_{F}, \rho_{Y}=1$, and $f(D)=Y$.

Proof. Suppose that $f(D) \subsetneq Y$. Then Lemma 3.9 says that $D=f^{-1}(f(D))$; in particular $D$ contains a fiber $F$, so

$$
\text { ker } f_{*}=\mathcal{N}_{1}(F, X) \subseteq \mathcal{N}_{1}(D, X)
$$

and $\rho_{X}-\rho_{Y} \leqslant 2$. If $\rho_{X}-\rho_{Y}=2$, then $\mathcal{N}_{1}(D, X)=\operatorname{ker} f_{*}$, so $D$ is a fiber and $Y \cong \mathbb{P}^{1}$. Thus we are in case (i).

If $\rho_{X}-\rho_{Y}=1$, then $f$ is elementary and $D$ is not a fiber, hence $\operatorname{dim} Y \geqslant 2$. If $\rho_{Y} \leqslant 3$, we are in case (ii).

Suppose that $\rho_{Y} \geqslant 4$. We have $\operatorname{dim} \mathcal{N}_{1}(f(D), Y)=1$, so Proposition 3.16 yields that $\operatorname{dim} Y=2$. Thus Theorem 1.1(i) gives case (iii).

Assume now that $f(D)=Y$. Then the restriction

$$
\left(f_{*}\right)_{\mid \mathcal{N}_{1}(D, X)}: \mathcal{N}_{1}(D, X) \longrightarrow \mathcal{N}_{1}(Y)
$$

is surjective, so $\rho_{Y} \leqslant 2$. If equality holds, then $\mathcal{N}_{1}(D, X) \cap \operatorname{ker} f_{*}=\{0\}$ and hence $\mathcal{N}_{1}(D, X) \cap$ $\operatorname{NE}(f)=\{0\}$. This implies that $f_{\mid D}$ is finite, so $f$ has one-dimensional fibers. Then $f$ is elementary and $\rho_{X}=3$, so we are again in case (ii). Finally if $\rho_{Y}=1$ we get case (iv).

Example 7.5 (Elementary contractions over surfaces). It is not difficult to write down examples of smooth Fano varieties of dimension $n \geqslant 3$ which are not products, but have an elementary contraction of fiber type over $\mathbb{P}^{2}, \mathbb{P}^{1} \times \mathbb{P}^{1}$, or $\mathbb{F}_{1}$. Thus the condition $\rho_{Y} \geqslant 3$ in the second part of Theorem 1.1(i) is necessary.

For instance, one can consider the $\mathbb{P}^{n-2}$-bundles

$$
\left.\mathbb{P}_{\mathbb{P}^{2}}\left(\mathcal{O}^{\oplus(n-2)} \oplus \mathcal{O}(1)\right), \quad \mathbb{P}_{\mathbb{P}^{1} \times \mathbb{P}^{1}}\left(\mathcal{O}^{\oplus(n-2)} \oplus \mathcal{O}(1,1)\right), \quad \mathbb{P}_{\mathbb{F}_{1}}\left(\mathcal{O}^{\oplus(n-2)} \oplus \mathcal{O}(l)\right)\right),
$$

where $l \subset \mathbb{F}_{1}$ is the proper transform of a general line in $\mathbb{P}^{2}$.

A different example is given by $\mathbb{F}_{1} \times \mathbb{P}^{1} \times \mathbb{P}^{n-3}$, which has a quasi-elementary contraction onto $\mathbb{P}^{1} \times \mathbb{P}^{1}$ with fiber $\mathbb{P}^{1} \times \mathbb{P}^{n-3}$. In this case the variety is a product, but it is not the product of the fiber and the target of the contraction.

Example 7.6 (Elementary contractions over 3-folds). Let $Y=\mathbb{P}_{\mathbb{P}^{1} \times \mathbb{P}^{1}}(\mathcal{O} \oplus \mathcal{O}(1,1)$ ), so that $Y$ is Fano with $\rho_{Y}=3$. Observe that $Y$ is the divisorial resolution of a quadric $Q \subset \mathbb{P}^{4}$ with an isolated singularity. Let $L \in \operatorname{Pic} Y$ be the pull-back of $\mathcal{O}_{Q}(1)$, and consider

$$
X=\mathbb{P}_{Y}\left(\mathcal{O}^{\oplus(n-3)} \oplus L\right) .
$$

Then $X$ is Fano with dimension $n$ and $\rho_{X}=4$, and it is not a product. One can write down analogous examples with $\rho_{Y}=1,2$.

We do not know whether there are similar examples with $\rho_{Y}=4,5$. Let us point out that smooth Fano 3-folds $Y$ with $\rho_{Y}=5$ (respectively $\rho_{Y}=4$ ) that are not products are given by just two families (respectively 12), after [MM81]. 


\section{Casagrande}

Example 7.7 (Conic bundles). Let $X$ be a smooth Fano variety and $f: X \rightarrow Y$ an equidimensional contraction with $\operatorname{dim} Y=n-1$. Then $f$ is a conic bundle, and it is quasi-elementary if and only if it is elementary.

We know that $Y$ is smooth (see [And85, Theorem 3.1] and [AW98, Proposition 4.1]) and if $\operatorname{dim} X \leqslant 4$ then $Y$ is also Fano, by [Wiś91a, Corollary on p. 156]. However, this is not true in higher dimensions, see [Wiś91a, Example on p. 156].

Example 7.8 (Elementary contractions in dimension 4). Let $X$ be a smooth Fano 4-fold and consider an elementary contraction $f: X \rightarrow Y$ with $\operatorname{dim} Y=3$.

If $f$ is equidimensional then $Y$ is smooth and Fano, see Example 7.7. However, it is well known that $f$ can have isolated two-dimensional fibers, which are classified, see [AW98, Kac97]. In [Kac97, $\S 11]$ we find several examples where $f$ is not equidimensional and $Y \cong \mathbb{P}^{3}$; in particular, in this case $X$ is not a product. However, we are not aware of similar examples with $Y$ singular.

Example 7.9 (A Fano 4-fold with $\rho=6$ and only small elementary contractions). In the toric case, smooth Fano varieties are classified up to dimension 7, the cases of dimensions 5, 6 and 7 being quite recent [KN07, Øbr07]. They are a good source of explicit examples.

After the classification in [Bat99] (see also [Sat00]), toric Fano 4-folds have Picard number at most 8. The ones with $\rho=7,8$ are just $S_{3} \times S_{3}$ and $S_{3} \times S_{2}, S_{2}$ and $S_{3}$ being the blow-up of $\mathbb{P}^{2}$ in two points and in three non-collinear points respectively. Among the ones with $\rho=6$ there is a case with no (non-trivial) quasi-elementary contractions, the toric Del Pezzo 4-fold $V$ (n. 118 in [Bat99]).

The Mori cone $\mathrm{NE}(V)$ has dimension 6 but has 20 extremal rays. Every elementary contraction is a small contraction with exceptional locus a $\mathbb{P}^{2}$ with normal bundle $\mathcal{O}_{\mathbb{P}^{2}}(-1)^{\oplus 2}$. Every such exceptional $\mathbb{P}^{2}$ intersects three others in a point.

One can see that $V$ has a contraction of fiber type $f: V \rightarrow Y$ with $\operatorname{dim} Y=3$ and $\rho_{Y}=1$, so $f$ is not quasi-elementary. There are six two-dimensional fibers, which are unions of two exceptional $\mathbb{P}^{2}$ intersecting in one point. Moreover, $Y$ has six isolated non- $\mathbb{Q}$-factorial points in the images of these fibers.

To our knowledge, among the known examples of Fano 4-folds with no (non-trivial) quasielementary contractions of fiber type, $V$ is the one with largest Picard number.

This example has an analog $V_{n}$ in each even dimension $n=2 m \geqslant 4$. This is a smooth toric Fano variety with $\rho_{V_{n}}=n+2$ and $2\left(\begin{array}{c}n+1 \\ m\end{array}\right)$ extremal rays. Every elementary contraction is a small contraction with exceptional locus a $\mathbb{P}^{m}$ with normal bundle $\mathcal{O}_{\mathbb{P}^{m}}(-1)^{\oplus m}$. The varieties $V_{n}$ are called toric Del Pezzo varieties and were introduced in [VK84].

\section{ACKNOWLEDGEMENTS}

I wish to thank Laurent Bonavero and Stéphane Druel for many discussions about Fano varieties and their Picard number, and about families of rational curves. My thanks also go to Rita Pardini, for her interest in this subject and for the conversations we had about it, and to Priska Jahnke, from whom I learnt a lot about singular Fano threefolds. Finally I thank the referee for useful remarks.

\section{REFERENCES}

ABW92 M. Andreatta, E. Ballico and J. A. Wiśniewski, Vector bundles and adjunction, Internat. J. Math. 3 (1992), 331-340.

ACO04 M. Andreatta, E. Chierici and G. Occhetta, Generalized Mukai conjecture for special Fano varieties, Cent. Eur. J. Math. 2 (2004), 272-293.

And85 T. Ando, On extremal rays of the higher dimensional varieties, Invent. Math. 81 (1985), 347-357. 


\section{QUASI-ELEMENTARY CONTRACTIONS OF FANO MANIFOLDS}

AW98 M. Andreatta and J. A. Wiśniewski, On contractions of smooth varieties, J. Algebraic Geom. 7 (1998), 253-312.

Bat99 V. V. Batyrev, On the classification of toric Fano 4-folds, J. Math. Sci. (New York) 94 (1999), 1021-1050.

BCD07 L. Bonavero, C. Casagrande and S. Druel, On covering and quasi-unsplit families of curves, J. Eur. Math. Soc. (JEMS) 9 (2007), 45-57.

BCDD03 L. Bonavero, C. Casagrande, O. Debarre and S. Druel, Sur une conjecture de Mukai, Comment. Math. Helv. 78 (2003), 601-626.

BCW02 L. Bonavero, F. Campana and J. A. Wiśniewski, Variétés projectives complexes dont l'éclatée en un point est de Fano, C. R. Acad. Sci. 334 (2002), 463-468.

Cam81 F. Campana, Coréduction algébrique d'un espace analytique faiblement Kählérien compact, Invent. Math. 63 (1981), 187-223.

Cam92 F. Campana, Connexité rationelle des variétés de Fano, Ann. Sci. École Norm. Sup. 25 (1992), $539-545$.

Cam04 F. Campana, Orbifolds, special varieties and classification theory: an appendix, Ann. Inst. Fourier (Grenoble) 54 (2004), 631-665.

Cas06 C. Casagrande, The number of vertices of a Fano polytope, Ann. Inst. Fourier (Grenoble) 56 (2006), 121-130.

Cut88 S. Cutkosky, Elementary contractions of Gorenstein threefolds, Math. Ann. 280 (1988), 521-525.

Deb01 O. Debarre, Higher-dimensional algebraic geometry, Universitext (Springer, Berlin, 2001).

Deb03 O. Debarre, Fano varieties, in Higher dimensional varieties and rational points, Budapest, 2001, Bolyai Society Mathematical Studies, vol. 12 (Springer, Berlin, 2003), pp. 93-132.

Ful93 W. Fulton, Introduction to toric varieties, Annals of Mathematics Studies, vol. 131 (Princeton University Press, Princeton, NJ, 1993).

FZ03 H. Flenner and M. Zaidenberg, Log-canonical forms and log canonical singularities, Math. Nachr. 254/255 (2003), 107-125.

IP99 V. A. Iskovskikh and Y. G. Prokhorov, Algebraic geometry V-Fano varieties, Encyclopaedia of Mathematical Sciences, vol. 47 (Springer, Berlin, 1999).

JR06 P. Jahnke and I. Radloff, Terminal Fano threefolds and their smoothings, Preprint (2006), arXiv:math/0601769.

Kac97 Y. Kachi, Extremal contractions from 4-dimensional manifolds to 3-folds, Ann. Scuola Norm. Sup. Pisa Cl. Sci. (4) 24 (1997), 63-131.

Kaw89 Y. Kawamata, Small contractions of four dimensional algebraic manifolds, Math. Ann. 284 (1989), 595-600.

KM98 J. Kollár and S. Mori, Birational geometry of algebraic varieties, Cambridge Tracts in Mathematics, vol. 134 (Cambridge University Press, Cambridge, 1998).

KMM87 Y. Kawamata, K. Matsuda and K. Matsuki, Introduction to the minimal model problem, in Algebraic geometry, Sendai, 1985, Advanced Studies in Pure Mathematics, vol. 10 (Mathematical Society of Japan, Tokyo, 1987), pp. 283-360.

KMM92 J. Kollár, Y. Miyaoka and S. Mori, Rational connectedness and boundedness of Fano manifolds, J. Differential Geom. 36 (1992), 765-779.

KN07 M. Kreuzer and B. Nill, Classification of toric Fano 5-folds, Preprint (2007), arXiv:math/0702890.

Kol86 J. Kollár, Higher direct images of dualizing sheaves I, Ann. of Math. (2) 123 (1986), 11-42.

Kol96 J. Kollár, Rational curves on algebraic varieties, Ergebnisse der Mathematik und ihrer Grenzgebiete, vol. 32 (Springer, Berlin, 1996).

Miy93 Y. Miyaoka, Relative deformations of morphisms and applications to fibre spaces, Comment. Math. Univ. S. Paul. 42 (1993), 1-7.

MM81 S. Mori and S. Mukai, Classification of Fano 3 -folds with $b_{2} \geqslant 2$, Manuscripta Math. 36 (1981), 147-162. Erratum: 110 (2003), 407. 


\section{QUASI-ELEMENTARY CONTRACTIONS OF FANO MANIFOLDS}

Nam97 Y. Namikawa, Smoothing Fano 3-folds, J. Algebraic Geom. 6 (1997), 307-324.

Øbr07 M. Øbro, An algorithm for the classification of smooth Fano polytopes, Preprint (2007), arXiv:0704.0049.

Pro05 Y. G. Prokhorov, On the degree of Fano threefolds with canonical Gorenstein singularities, Mat. Sb. 196 (2005), 81-122 (in Russian). English translation: Sb. Math. 196 (2005), 77-114.

Sar82 V. G. Sarkisov, On conic bundle structures, Izv. Akad. Nauk SSSR, Ser. Mat. 46 (1982), 371-408 (in Russian). English translation: Math. USSR Izv. 20 (1982), 355-390.

Sat00 H. Sato, Toward the classification of higher-dimensional toric Fano varieties, Tôhoku Math. J. (2) $\mathbf{5 2}$ (2000), 383-413.

Tsu06 T. Tsukioka, Classification of Fano manifolds containing a negative divisor isomorphic to projective space, Geom. Dedicata 123 (2006), 179-186.

VK84 V. E. Voskresenskiü and A. Klyachko, Toric Fano varieties and systems of roots, Izv. Akad. Nauk SSSR, Ser. Mat. 48 (1984), 237-263 (in Russian). English translation: Math. USSR Izv. 24 (1985), 221-244.

Voi02 C. Voisin, Théorie de Hodge et géométrie algébrique complexe, Cours Spécialisés, vol. 10 (Société Mathématique de France, Paris, 2002).

Wat80 K. Watanabe, On plurigenera of normal isolated singularities. I, Math. Ann. 250 (1980), 65-94.

Wiś91a J. A. Wiśniewski, On contractions of extremal rays of Fano manifolds, J. reine angew. Math. 417 (1991), 141-157.

Wiś91b J. A. Wiśniewski, On deformation of nef values, Duke Math. J. 64 (1991), 325-332.

Cinzia Casagrande cinzia.casagrande@unipv.it

Dipartimento di Matematica, Università di Pisa, Largo B. Pontecorvo 5, 56127 Pisa, Italy

Current address: Dipartimento di Matematica, Università di Pavia, Via Ferrata 1, 27100 Pavia, Italy 